University of Nebraska - Lincoln

DigitalCommons@University of Nebraska - Lincoln

2012

\title{
A reaction progress variable modeling approach for non-ideal multiphase explosives
}

\author{
K. P. Ruggirello \\ University at Buffalo, Buffalo, NY \\ P. E. DesJardin \\ University at Buffalo, Buffalo, NY \\ M. R. Baer \\ Sandia National Laboratories, Albuquerque, NM \\ M. J. Kaneshige \\ Sandia National Laboratories, Albuquerque, NM \\ E. S. Hertel \\ Sandia National Laboratories, Albuquerque, NM
}

Follow this and additional works at: https://digitalcommons.unl.edu/usdoepub

Part of the Bioresource and Agricultural Engineering Commons

Ruggirello, K. P.; DesJardin, P. E.; Baer, M. R.; Kaneshige, M. J.; and Hertel, E. S., "A reaction progress variable modeling approach for non-ideal multiphase explosives" (2012). US Department of Energy Publications. 130.

https://digitalcommons.unl.edu/usdoepub/130

This Article is brought to you for free and open access by the U.S. Department of Energy at DigitalCommons@University of Nebraska - Lincoln. It has been accepted for inclusion in US Department of Energy Publications by an authorized administrator of DigitalCommons@University of Nebraska - Lincoln. 


\title{
A reaction progress variable modeling approach for non-ideal multiphase explosives
}

\author{
K.P. Ruggirello ${ }^{\text {a }}$, P.E. DesJardin ${ }^{\mathrm{a}, *}$, M.R. Baer ${ }^{\mathrm{b}}$, M.J. Kaneshige ${ }^{\mathrm{b}}$, E.S. Hertel ${ }^{\mathrm{b}}$ \\ ${ }^{a}$ Department of Mechanical and Aerospace Engineering, University at Buffalo, Buffalo, NY 14228, USA \\ ${ }^{\mathrm{b}}$ Sandia National Laboratories, Albuquerque, NM 87123, USA
}

\section{A R T I C L E I N F O}

\section{Article history:}

Received 28 September 2011

Received in revised form 7 February 2012

Accepted 8 February 2012

Available online 24 February 2012

\section{Keywords:}

Explosives

Shocks

Aluminum

Compressible flow

\begin{abstract}
A B S T R A C T
This study concerns the development of a mixture fraction based reaction progress variable formulation for aluminized explosives. Highlights of the formulation include a fully compressible treatment of both the gas and solid phases (both aluminum and alumina), heterogenous and homogenous reactions, and effects of group combustion. Isolated particle simulations are validated against experimental data and DNS and show good agreement of burn times over a range of pressure and oxygen environments. The new models are implemented in the CTH shock physics code using a fractional step approach to allow for efficient computation of particle dynamics. Comparisons are made to experimental pressure data for a thermobaric explosive in the Sandia Explosive Components Facility (ECF). Parametric studies are conducted to determine pressure response and impulse to charge equivalence ratio and particle size. Overall good agreement is observed between simulation predictions of pressure time history and impulse.
\end{abstract}

(c) 2012 Elsevier Ltd. All rights reserved.

\section{Introduction}

While the behavior of ideal explosives is well understood and mature scaling theories are established (Cooper, 1996), much less is known about non-ideal multiphase explosives. In an ideal explosive, the effects of reactions can be explained by well established Chapman-Jouget $(\mathrm{CJ})$ theory for detonations and the resulting expansion process can be explained by non-reacting gas dynamics. Non-ideal explosives, however, do not follow the same well defined detonation jump relations and therefore significant deviations are expected for the CJ pressure, velocity, or expansion isentrope predicted from equilibrium, steady-state calculations such as those typically used in BKW (Mader, 1998), TIGER (Cowperthwaite, 1973), and CHEETAH (Fried and Souers, 1994). The observed detonation pressures can be hundreds of kilobars below the predicted steady-state calculations (Orth and Krier, 1998). Nonideal explosives also show increased sensitivity to confinement, diameter, and oxidizing environment which are all controlled by local turbulent mixing processes. Additionally they can have reaction zones which are on the order of centimeters rather than microns found in ideal explosives (Jackson et al., 2011), and have delayed reactions that take place in the expansion wave which support the air shock. The reactions in the expansion wave of a non-ideal explosive occur as both anaerobic from reactions with the detonation products, and aerobic reactions from mixing with surrounding oxidants such as oxygen in the air. Even though the

\footnotetext{
* Corresponding author. Tel.: +1 7166451467.

E-mail address: ped3@buffalo.edu (P.E. DesJardin).
}

detonation pressures are lower, the detonation wave from nonideal explosives have wider pressure profiles which leads to an increased impulse $\left(I=\int p d t\right)$, as illustrated in Fig. 1 .

Non-ideal multiphase explosives do not have the fuel and oxidizer mixed on a molecular level, and usually a fine reactive metal powder such as aluminum is added to a mixture of high explosive(s), and binder. The metallic powders nominal diameter has a significant effect on the detonation behavior and is typically of the order of 10 s of microns to nanometers in size in more recent compositions, where the smaller particle size is desirable due to lower thermal inertia and increased reaction surface area. The shape of the particles also has an effect on the explosive properties, where metallic flakes are typically used to further increase the surface area for reactions. This metalized high explosive mixture is then placed around a booster charge which serves to initiate the explosive and also to disperse the metallic fuel to the surrounding atmosphere where it may use the excess oxygen if the mixture is fuel rich.

In an effort to improve the predictions made by the equilibrium thermodynamics codes Keshavarz et al. (2006) recently developed a simple empirical relationship to predict the detonation pressure for a general $\mathrm{C}_{a} \mathrm{H}_{b} \mathrm{~N}_{c} \mathrm{O}_{d} \mathrm{Al}_{e}$ non-ideal explosive, but more advanced physics based models for the prediction of the detonation properties of non-ideal explosives are still lacking. The focus of this study is to explore the ignition and burning of aluminum particles in a multiphase high pressure and temperature shock environment following the detonation of a non-ideal aluminized high explosive (TBX) where both anaerobic and aerobic reactions occur. 


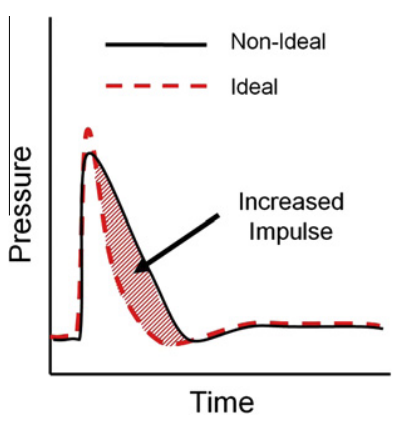

Fig. 1. Representative pressure profile for an ideal vs. non-ideal explosive.

Early semi-analytical theoretical models of aluminum combustion have focused on the quasi-steady burning stages (Brzustowski and Glassman, 1964; Law, 1973; Turns et al., 1987). These models employ flame sheet approximations and decompose the transport of heat and mass into two zones: a region between the particle surface and the flame, and a region beyond the flame. Results using these models have been shown to provide reasonable predictions for burning rates in a variety of oxidizing environments (Turns et al., 1987; Brooks and Beckstead, 1995). Beckstead et al. investigated a more detailed description of the flame and flow around the particle but found that many of the aspects of the flame structure and overall burning rates are close to those obtained using flame sheet assumptions (Liang and Beckstead, 1998; Widener et al., 1998). Babuk and Vasilyev have devised a five zone model that includes a more complete description of oxide cap formation, growth and movement (Babuk and Vasilyev, 2002). They demonstrate that their model is capable of capturing many of the observed dynamics of agglomerate motion. Most recently, Washburn et al. (2008, 2010) have combined the Liang/Beckstead (Liang and Beckstead, 1998; Widener et al., 1998) model with a detailed chemical-kinetics mechanism and direct numerical simulation (DNS) of the gas phase around the particle to examine the combustion characteristics for a range of oxidizing and pressure environments. In the current study a previously developed aluminum particle combustion model (DesJardin et al., 2005; Ruggirello et al., 2010) is used which relies on Shvab-Zel'dovich coupling functions (Kuo, 1986) to efficiently solve the coupled heat and mass transfer for the particle.

In order to extend the single particle model to a reactive particulate cloud consisting of potentially millions of particles, a multiphase model is used in the current study to solve the solid and gas phases along with their interactions. Multiphase flow theory has an extensive background and range of applications including fluidized beds (Mathiesen et al., 2000; Samuelsberg and Hjertager, 1996), powder compaction (Saurel et al., 2010), and deflagration to detonation transition in granular materials (Baer and Nunziato, 1986; Bdzil et al., 1999; Kapila et al., 2001). The majority of the multiphase models are based off the two-phase mixture model developed by Baer and Nunziato (1986). It allows for disequilibrium of pressures, velocities, and temperatures between the phases and uses the second law of thermodynamics to construct admissible phase interaction terms. There are several challenges in multiphase flow modeling, which are outlined by Bdzil et al. (1999). The phase interaction terms lead to non-conservative governing equations for each phase and the very short time scales $\left(\approx 10^{-8} \mathrm{~s}\right)$ associated with the equilibrium processes make the equations very stiff. In an effort to alleviate the stiffness several authors have developed reduced equation models which assume a single pressure (Paillre et al., 2003; Liou et al., 2008; Chang and Liou, 2007), a single velocity, or a single velocity and pressure (Kapila et al., 2001). When a single pressure is assumed between the phases the hyperbolic nature of the equations is lost, and a pressure correction term is usually added to the interface pressure to restore hyperbolicity (Stuhmiller, 1977; Chang and Liou, 2007; Liou et al., 2008). Another method to reduce the stiffness of the equations is by using a pressure relaxation method. The pressure relaxation methods subcyle the pressure work term between the phases separately from the hydrodynamics by adjusting the volume fractions until mechanical equilibrium is reached. There are several different pressure relaxation algorithms presented in the literature (Saurel and Abgrall, 1999; Chinnayya et al., 2004; Petitpas et al., 2009; Saurel et al., 2009; Benson, 1992; Lallemand et al., 2005). In this study the multiphase model of Baer and Nunziato (1986) is used to model the solid and gas phases and a pressure relaxation method based on the algorithm presented by Saurel et al. (2009) is used to alleviate the stiffness of the equations.

To recast the previously developed Lagrangian aluminum particle combustion model (DesJardin et al., 2005; Ruggirello et al., 2010) into an Eulerian framework, a reaction progress variable description is used. An Eulerian framework for the aluminum particles is chosen because of the desire to account for the group combustion burning mode for aluminum rich charges. Reaction progress variable approaches have been used in non-premixed turbulent combustion (Pitsch et al., 2003; Bray et al., 2005; Pitsch and Ihme, 2005) to reduce the degrees of freedom and account for subgrid scale (SGS) turbulence and combustion. The non-premixed nature of the multiphase aluminum particle combustion makes the reaction progress variable formulation an attractive modeling approach for this study.

The model presented is unique in that it combines a detailed mechanistic aluminum particle combustion model with a reaction progress variable formulation and a multiphase flow model. The combination of these allows the dynamics of the aluminum particle combustion, and the group combustion burning mode to be simulated. Additionally the multiphase flow model allows the phase interactions between the aluminum/alumina and gas products to be explicitly accounted for.

The remainder of this paper is organized as follows. First the two-phase flow model is presented, followed by the aluminum particle combustion model and reaction progress variable formulation. Next the numerical implementation is discussed, and results are presented. The results consist of isolated single particle cases, experimental comparisons to several tests conducted at the Explosives Components Facility (ECF) at Sandia National Laboratories, and sensitivity studies conducted to determine the effects of initial particle diameter and equivalence ratio on the model. Finally, conclusions are drawn from this study.

\section{Mathematical formulation}

\subsection{Two-phase flow model}

The multiphase system is formulated by phase-averaging of the instantaneous multiphase equations over a representative volume that is compactly defined by the filter function, $G\left(\boldsymbol{x}-\boldsymbol{x}^{\prime}\right)$, with the normalization property, $\int_{V} G d V=1$ (Carrara and DesJardin, 2006). Application of the filtering operator and neglecting bulk phase molecular viscosity, conduction and diffusion processes results in the following system of equations for volume, mass, momentum and energy transport:

$$
\begin{aligned}
& \frac{\partial \alpha_{k}}{\partial t}+\boldsymbol{u}_{k} \cdot \nabla \alpha_{k}=V_{k}^{\ddagger}+c_{k}^{\ddagger} / \gamma_{k} \\
& \frac{\partial \rho_{k}}{\partial t}+\nabla \cdot\left(\rho_{k} \boldsymbol{u}_{k}\right)=c_{k}^{\ddagger} \\
& \frac{\partial \rho_{i, k}}{\partial t}+\nabla \cdot\left(\rho_{i, k} \boldsymbol{u}_{k}\right)=\alpha_{k} \dot{m}_{k, i}^{\prime \prime \prime}+c_{k, i}^{\ddagger}
\end{aligned}
$$


$\frac{\partial\left(\rho_{k} \boldsymbol{u}_{k}\right)}{\partial t}+\nabla \cdot\left(\rho_{k} \boldsymbol{u}_{k} \boldsymbol{u}_{k}\right)=-\nabla \cdot\left(\alpha_{k} p_{k}\right)+\boldsymbol{m}_{k}^{\ddagger}$

$\frac{\partial\left(\rho_{k} E_{k}\right)}{\partial t}+\nabla \cdot\left(\rho_{k} \boldsymbol{u}_{k} E_{k}\right)=-\nabla \cdot\left(\alpha_{k} p_{k} \boldsymbol{u}_{k}\right)+e_{k}^{\ddagger}$

where the subscript $k$ represents a given phase (or material) of the multiphase (-material) system, $\rho_{k}=\alpha_{k} \gamma_{k}$ is the material partial density, $\alpha_{k}$ is the volume fraction and $\gamma_{k}$ is the true material density. In Eq. (1c) $\rho_{i, k}=\alpha_{k} \gamma_{k, i}$ is the density of the $i$ th species constituent of the $k$ th phase (or material) and $\dot{m}_{k, i}^{\prime \prime \prime}$ is the production or consumption of species from homogeneous reactions. The quantity $E_{k}=\sum e_{k, i} \rho_{i, k} /$ $\rho_{k}+\boldsymbol{u}_{k} \cdot \boldsymbol{u}_{k} / 2$ in Eq. (1e) is the total energy per unit mass of the $k$ th phase and $e_{k, i}$ is the energy per unit mass of the $i$ th species. The terms $V_{k}^{\ddagger}, c_{k, i}^{\ddagger}, c_{k}^{\ddagger}, \boldsymbol{m}_{k}^{\ddagger}$ and $e_{k}^{\ddagger}$ represent source terms for volume (compaction), mass, momentum and energy phase-exchange processes and are defined as,

$V_{k}^{\ddagger}=\int_{\partial \Omega_{k}} \boldsymbol{u}_{k}^{\prime} \cdot \boldsymbol{n}_{k} G d A_{k}$

$c_{k, i}^{\ddagger}=\int_{\partial \Omega_{k}} \dot{m}_{k, i}^{\prime \prime} G d A_{k}$

$c_{k}^{\ddagger}=\sum_{i=1}^{N} c_{k, i}^{+}=\int_{\partial \Omega_{k}} \dot{m}_{k}^{\prime \prime} G d A_{k}$

$\boldsymbol{m}_{k}^{\ddagger}=\int_{\partial \Omega_{k}}\left(\boldsymbol{u}_{k} \dot{m}_{k}^{\prime \prime}+\underset{\sim}{\sigma_{k}} \cdot \boldsymbol{n}_{k}\right) G d A_{k}$

$e_{k}^{\ddagger}=\int_{\partial \Omega_{k}}\left[\sum_{i} e_{k, i} \dot{m}_{k, i}^{\prime \prime}+\dot{m}_{k}^{\prime \prime} \boldsymbol{u}_{k} \cdot \boldsymbol{u}_{k} / 2-\dot{q}_{k}^{\prime \prime}+\left(\boldsymbol{u}_{k} \cdot \boldsymbol{\sigma}_{\sim}\right) \cdot \boldsymbol{n}_{k}\right] G d A_{k}$

where $\dot{m}_{k, i}^{\prime \prime}\left(=\gamma_{k, i}\left(\boldsymbol{V}_{k}^{I}-\boldsymbol{u}_{k, i}\right) \cdot \boldsymbol{n}_{k}\right)$ and $\dot{m}_{k}^{\prime \prime}\left(=\gamma_{k}\left(\boldsymbol{V}_{k}^{I}-\boldsymbol{u}_{k}\right) \cdot \boldsymbol{n}_{k}\right)$ are the mass fluxes for the $i$ th species and total mass fluxes per unit surface area across the phase boundary $\left(\partial \Omega_{k}\right)$ defined in terms of the phase interface velocity $\left(\boldsymbol{V}_{k}^{I}\right)$ and phase boundary surface area normal $\left(\boldsymbol{n}_{k}\right)$. The quantity, $\boldsymbol{u}_{k}^{\prime}$ in Eq. (2a) represents the fluctuations of the $k$ th material velocity from the mean and comes from a linearization of the phase-averaged volume fraction advection term (see Chinnayya et al. (2004) for details). The quantities $\dot{q}_{k}^{\prime \prime}=-k_{k} \nabla T \cdot \boldsymbol{n}_{k}$ and $\boldsymbol{\sigma}_{\boldsymbol{k}}$ are the heat conduction and Cauchy stress at the surface of the phase boundary. In order to satisfy total mixture volume, mass, momentum and energy conservation the phase-exchange terms must sum to zero, $\sum_{k} V_{k}^{\ddagger}=0$ and $\sum_{k} \alpha_{k}=1, \sum_{k} c_{k}^{\ddagger}=0, \sum_{k} \boldsymbol{m}_{k}^{\ddagger}=0$ and $\sum_{k} e_{k}^{\ddagger}=0$.

Alternatively, the conservation equations of Eq. (1) may be expressed in terms of a Lagrangian reference frame with a mixture weighted velocity that is useful for numerical implementation into existing hydrocodes that are based on multi-material descriptions of the governing equations (McGlaun et al., 1990; van Leer, 1979). To recast the Eulerian conservation equations into a mixture material Lagrangian reference frame, the mixture weighted material derivative is first defined as $\dot{\Theta}_{k}=\frac{\partial \Theta_{k}}{\partial t}+\boldsymbol{u} \cdot \nabla \Theta_{k}$, where $\boldsymbol{u}=\sum_{k} \rho_{k} \boldsymbol{u}_{k} / \rho$ is the mass weighted mixture velocity. The conservation equations for each phase can then be expressed as (Baer and Nunziato, 1986),

$\dot{\alpha}_{k}=\left(\boldsymbol{D}_{k} / \rho_{k}\right) \cdot \nabla \alpha_{k}+V_{k}^{\ddagger}+c_{k}^{\ddagger} / \gamma_{k}$

$\dot{\rho}_{k}=\boldsymbol{D}_{k} \cdot \nabla \rho_{k}-\rho_{k} \nabla \cdot \boldsymbol{u}_{k}+c_{k}^{\ddagger}$

$\dot{\rho}_{i, k}=\boldsymbol{D}_{k} \cdot \nabla \rho_{i, k}-\rho_{i, k} \nabla \cdot \boldsymbol{u}_{k}+\alpha_{k} \dot{m}_{k, i}^{\prime \prime \prime}+c_{k, i}^{\ddagger}$

$\rho_{k} \dot{\boldsymbol{u}}_{k}=\boldsymbol{D}_{k} \cdot \nabla \boldsymbol{u}_{k}-\nabla\left(\alpha_{k} p_{k}\right)+\boldsymbol{m}_{k}^{\ddagger}-c_{k}^{\ddagger} \boldsymbol{u}_{k}$

$$
\begin{aligned}
\rho_{k} \dot{e}_{k}= & \boldsymbol{D}_{k} \cdot \nabla e_{k}-\alpha_{k} p_{k} \nabla \cdot \boldsymbol{u}_{k} \\
& +e_{k}^{\ddagger}-\boldsymbol{m}_{k}^{\ddagger} \cdot \boldsymbol{u}_{k}-c_{k}^{\ddagger}\left(e_{k}-\boldsymbol{u}_{k} \cdot \boldsymbol{u}_{k} / 2\right)
\end{aligned}
$$

where the first term on the r.h.s. containing the quantity, $\boldsymbol{D}_{k} \equiv \rho_{k}\left(\boldsymbol{u}-\boldsymbol{u}_{k}\right)$, is the drift term and represents the effects from the relative slip velocity between phase $k$ and that of the mixture.

A fundamental axiom of continuum mixture theory is that the overall conservation relationships are assumed to be preserved for all possible conditions. Thus, the conservation equations for the total mixture are defined for a homogeneous material by summing the individual constituent conservation equations. Carrying out this summation produces additional mixture constraints that define a mixture pressure, deviatoric stress and mixture energy:

$$
\begin{aligned}
& p+\rho \boldsymbol{u} \cdot \boldsymbol{u}=\sum_{k}\left(\alpha_{k} p_{k}+\rho_{k} \boldsymbol{u}_{k} \cdot \boldsymbol{u}_{k}\right) \\
& \underset{\sim}{\tau}=\sum_{k} \alpha_{k} \underset{\sim_{k}}{\tau} \\
& \rho(e+\boldsymbol{u} \cdot \boldsymbol{u} / 2)=\sum \rho_{k}\left(e_{k}+\boldsymbol{u}_{k} \cdot \boldsymbol{u}_{k} / 2\right) .
\end{aligned}
$$

As shown by Baer and Nunziato (1986), by using these constraints and summing conservation Eqs. (3b), (3d) and (3e) over all phases recovers the overall conservation equations that are identical to those for a single phase flow (ignoring bulk viscous and diffusion processes).

$\dot{\rho}=-\rho \nabla \cdot \boldsymbol{u}$

$\rho \dot{u}=-\nabla p$

$\rho \dot{e}=-p \nabla \cdot \boldsymbol{u}$

where the following mixture weighted properties are employed:

$\rho=\sum_{k} \alpha_{k} \rho_{k}$

$p=\sum_{k} \alpha_{k} p_{k}$

$e=\sum_{k} \rho_{k} e_{k} / \rho$

Summing over all phases for Eq. (3c), however, results in the following result:

$\rho \dot{Y}_{i}=\nabla \cdot\left(Y_{i} \boldsymbol{D}_{i}\right)+\dot{m}_{i}^{\prime \prime \prime}+c_{i}$

where $Y_{i}$ is the mass ratio of the $i$ th species and the total mixture mass and $\dot{m}_{i}^{\prime \prime \prime}$ and $c_{i}$ represent the effects from bulk (mixture) homogeneous and heterogeneous reactions, respectively, defined as,

$Y_{i}=\sum_{k} \rho_{k, i} / \rho$

$\dot{m}_{i}^{\prime \prime \prime}=\sum_{k} \alpha_{k} \dot{m}_{i, k}^{\prime \prime \prime}$

$c_{i}=\sum_{k} c_{k, i}^{\ddagger}$.

In Eq. (7), $\boldsymbol{D}_{i} \equiv \rho\left(\boldsymbol{u}-\boldsymbol{u}_{i}\right)$ is a drift term that represents the effects from the relative slip velocity between the $i$ th phase $\left(\boldsymbol{u}_{i} \equiv \sum_{k} \boldsymbol{u}_{k} Y_{k, i} / Y_{i}\right)$ and that of the multiphase mixture. It is therefore apparent that the equivalent single phase conservation equation for the $i$ th constituent (i.e., $\rho \dot{Y}_{i}=\dot{m}_{i}^{\prime \prime \prime}+\dot{c}_{i}$ ) is not recovered when the individual phase equations are summed and a drift term is persistent in the final result. The appearance of the drift term posses challenges with regard to unique definitions for the reaction progress variable description, as will be discussed in Section 2.3.

The set of equations in (3) comprise $K(6+N-1)$ independent equations in 3D where $N$ is the total number of species constituents and $K$ is the total number of phases (or materials). With the specification of an equation of state (EOS) for each phase (or material) and closure of the phase coupling and reactions source terms 
(to be discussed) then a complete description of the multiphase system is defined. Alternatively, $K(6+N-1)-1$ material equations may be solved in addition to Eqs. (5) and (7) defining the overall mixture conservation. The latter approach is pursued in this study. The advantage of solving for the mixture conservation equations is twofold. The first is to guarantee overall conservation properties are explicitly enforced. The second is the ability to enforce local constraints in the mixture instead of solving separate transport equations. Examples include imposing local pressure or temperature equilibrium among phases (or materials) thereby eliminating the need to solve separate momentum or energy transport equations, respectively.

For the current study, the multiphase system is described as a collection of reactive particulate and gases. Each aluminum particle is assumed to be composed of two phases of aluminum (liquid and solid) and a secondary material, aluminum oxide (alumina) that forms a cap on the particle. All phases and materials that make up the particle are assumed to be in mechanical and thermal equilibrium (i.e., pressure and temperature are assumed equal) therefore only a single set of conservations equations are required for the particulate phase. In addition, there is fine alumina (i.e., nanoscale in size) produced in the diffusion flame surrounding the molten aluminum droplet from condensation reactions - similar to soot formation in hydrocarbon droplets. Some of the fine alumina diffuses back to the surface of the droplet and adds to the cap. The remainder diffuses to the "far-field" and mixes with the gas. This fine alumina is assumed to be in thermal and mechanical equilibrium with the particles thereby avoiding the need to solve separate transport equations for that phase. The gas phase system is assumed to consist of a mixture of reactive gases that obey the mixing rules defined by the Becker-Kistiakowsky-Wilson (BKW) equation of state (EOS) (Mader, 1998), therefore only a single set of gas phase conservation equations are needed. More details on the definition of the gas-phase EOS and the specific species considered are provided in Section 3. It should be emphasized that the particles and gas phases are not assumed to be in either temperature or pressure equilibrium. The rate for which these phases equilibrate is determined by the phase interaction terms which are defined in detail in Section 2.2.

The system of equations describing the reacting particulate phase can be further simplified if the effects of agglomeration and coagulation are neglected. In this limit, the phase interaction terms of Eq. (2) can be simplified to:

$\boldsymbol{S}_{s}^{\ddagger} \simeq \rho n_{P} \boldsymbol{S}_{P}^{\ddagger}$

where the subscript $P$ denotes a particle property, $n_{P}\left(=Y_{P} / m_{P}\right.$, where $m_{P}$ is the mass of a particle) is the particle number density per unit mass of the mixture, $\rho$ is the mixture density, $\boldsymbol{S}_{s}^{\ddagger}\left(=\left\{V_{s}^{\ddagger}, c_{s, i}^{\ddagger}, c_{s}^{\ddagger}, \boldsymbol{m}_{s}^{\ddagger}, e_{s}^{\ddagger}\right\}\right)$ is the vector of source terms for the entire "solid" particulate phase (which may contain liquids as well) and $\boldsymbol{S}_{P}^{\ddagger}$ are the respective source terms defined on a particle level (to be discussed further in Section 2.2) and are defined as,

$$
\begin{aligned}
V_{P}^{\ddagger} & =\int \boldsymbol{u}_{P}^{\prime} \cdot \boldsymbol{n}_{P} d A_{P} \\
c_{P}^{\ddagger} & =\int \dot{m}_{P}^{\prime \prime} d A_{P} \\
c_{i_{P}}^{\ddagger} & =\int \dot{m}_{P, i}^{\prime \prime} d A_{P} \\
\boldsymbol{m}_{P}^{\ddagger} & =\int \boldsymbol{u}_{P} \dot{m}_{P}^{\prime \prime}+\underset{\sim P}{\boldsymbol{\sigma}} \cdot \boldsymbol{n}_{P} d A_{P} \\
e_{P}^{\ddagger} & =\int \sum_{i} e_{P, i} \dot{m}_{P, i}^{\prime \prime}+\dot{m}_{P}^{\prime \prime} \boldsymbol{u}_{P} \cdot \boldsymbol{u}_{P} / 2-\dot{q}_{P}^{\prime \prime}+\left(\boldsymbol{u}_{P} \cdot \boldsymbol{\sigma}_{P}\right) \cdot \boldsymbol{n}_{P} d A_{P}
\end{aligned}
$$

Substituting the mass fraction definitions: $Y_{k} \equiv \rho_{k} \mid \rho$ and $Y_{i, k} \equiv \rho_{i, k} \mid \rho$ into Eq. (3) and using Eq. (5a) then the particle phase system of equations can be simplified to the following for the solid phase $(s)$ associated with the particulate:

$\dot{\alpha}_{s}=\left(\boldsymbol{D}_{s} / \rho_{s}\right) \cdot \nabla \alpha_{s}+\rho n_{P}\left(V_{P}^{\ddagger}+c_{P}^{\ddagger} / \gamma_{P}\right)$

$\dot{Y}_{s}=\left(\nabla \cdot \boldsymbol{D}_{s}\right) / \rho+n_{P} C_{P}^{\ddagger}$

$\dot{Y}_{s, i}=\left(\nabla \cdot \boldsymbol{D}_{s}\right) / \rho+\alpha_{s} \dot{m}_{s, i}^{\prime \prime \prime} / \rho+n_{P} C_{P, i}^{\ddagger}$

$\dot{\boldsymbol{u}}_{s}=\left(\boldsymbol{D}_{s} \cdot \nabla \boldsymbol{u}_{s}\right) /\left(\rho Y_{s}\right)-\alpha_{s} \nabla p_{s} /\left(\rho Y_{s}\right)+\left[\boldsymbol{m}_{P}^{\ddagger}-c_{P}^{\ddagger} \boldsymbol{u}_{s}\right] n_{P} / Y_{s}$

$\dot{e}_{s}=\left(\boldsymbol{D}_{s} \cdot \nabla e_{s}\right) /\left(\rho Y_{s}\right)-\alpha_{s} p_{s} \nabla \cdot \boldsymbol{u}_{s} /\left(\rho Y_{s}\right)$

$$
+\left[e_{P}^{\ddagger}-\boldsymbol{m}_{P}^{\ddagger} \cdot \boldsymbol{u}_{s}-c_{P}^{\ddagger}\left(e_{s}-\boldsymbol{u}_{s} \cdot \boldsymbol{u}_{s} / 2\right)\right] n_{P} / Y_{s}
$$

Furthermore the last term in Eqs. (11a), (11d) and (11e) may be simplified further by decomposing the particle surface stress and velocity components into a mean solid and fluctuating components associated with changes around the surface of the particle,

$\underset{\sim p}{\boldsymbol{\sigma}}=\underset{\sim s}{\boldsymbol{\sigma}}+\underset{\sim p}{\boldsymbol{\sigma}^{\prime}}$

$\boldsymbol{u}_{P}=\boldsymbol{u}_{s}+\boldsymbol{u}_{P}^{\prime}$

Substituting these relations into Eqs. (10d) and (10e) results in the following set of relations:

$$
\begin{aligned}
\boldsymbol{m}_{P}^{\ddagger}= & \boldsymbol{u}_{s} c_{p}^{\ddagger}+\int \underset{\sim_{p}}{\boldsymbol{\sigma}^{\prime}} \cdot \boldsymbol{n}_{p} d A_{p}+\underset{\sim_{s}}{\boldsymbol{\sigma}} \cdot \int \boldsymbol{n}_{p} d A p-\int \boldsymbol{u}_{p}^{\prime} \dot{m}_{p}^{\prime \prime} d A_{p} \\
\boldsymbol{e}_{P}^{\ddagger}= & c_{p}^{\ddagger}\left(e_{s}+\boldsymbol{u}_{s} \cdot \boldsymbol{u}_{s} / 2\right)+\boldsymbol{u}_{s} \cdot \int \underset{\sim_{s}}{\boldsymbol{\sigma}} \cdot \boldsymbol{n}_{p} d A_{p}-\int \boldsymbol{q}_{p}^{\prime \prime} d A_{p} \\
& +\int\left(\underset{\sim_{s}}{\boldsymbol{\sigma}} \cdot \boldsymbol{u}_{p}^{\prime}\right) \cdot \boldsymbol{n}_{p} d A_{p}+\left(\boldsymbol{u}_{s} \cdot \underset{\sim_{s}}{\boldsymbol{\sigma}}\right) \cdot \int \boldsymbol{n}_{p} d A_{p} \\
& +\int\left(\underset{\sim p}{\boldsymbol{\sigma}} \cdot \boldsymbol{u}_{p}^{\prime}\right) \cdot \boldsymbol{n}_{p} d A_{p}
\end{aligned}
$$

where the last two terms in Eqs. (13a) and (13b) are either identically zero since $\int \boldsymbol{n}_{p} d A_{p}=0$ for any closed surface or may be considered negligible - involving the products of fluctuations. The second terms on the r.h.s. involving the integration of the fluctuation of the Cauchy stress terms representing the effects of drag forces and associated rate of work on the particle. The third term on the r.h.s. of Eq. (13b) is the net heat transfer into the particle. Finally, the fourth term on the r.h.s. of Eq. (13b) is associated with the rate of compression or expansion work on the particle and may be simplified by assuming $\underset{\sim \varsigma}{\boldsymbol{\sigma}} \simeq-p_{I, P} \underset{\sim}{\boldsymbol{I}}$, where $p_{I, P}$ is the average interface pressure on the particle and $\boldsymbol{I}$ is the identity matrix. The interface pressure can be further expressed in terms of a mean particle pressure $\left(p_{P}\right)$ and a configurational stress $\left(\beta_{P}\right)$, i.e., $p_{I, P}=p_{P}-\beta_{P}$. Substituting these relations into the fourth term on the r.h.s. of Eq. (13b) results in,

$\int\left(\underset{\sim_{s}}{\boldsymbol{\sigma}} \cdot \boldsymbol{u}_{P}^{\prime}\right) \cdot \boldsymbol{n}_{P} d A_{P}=-\left(p_{P}-\beta_{P}\right) \int \boldsymbol{u}_{P}^{\prime} \cdot \boldsymbol{n}_{P} d A_{P}=-\left(p_{P}-\beta_{P}\right) V_{P}^{\ddagger}$.

In a previous study by Ruggirello et al. (2010) this term is modeled as: $\int \boldsymbol{u}_{P}^{\prime} \cdot \boldsymbol{n}_{P} d A_{P} \simeq V_{P} \alpha_{g}\left[p_{P}-p_{g}-\beta_{P}\right] / \mu_{c}$, where $\beta_{P}$ and $\mu_{c}$ are defined as the configurational pressure and $\mu_{c}$ is the compaction viscosity (Ruggirello et al., 2010). This model is a direct particle model analog to the compaction model of Baer and Nunziato developed for mixtures. In their study, the functional form for the compaction model came from an entropy based inequality constraint in the context of rational thermodynamics (Baer and Nunziato, 1986). The purpose of this model is to account for the bulk effect of unresolved mesoscale 
wave mechanics that serve to equilibrate the solid and gas phases. The time scale for pressure relaxation within the particle $(\tau)$ may be estimated as: $\tau=N D_{P} / c_{P}$, where $D_{P}$ is the particle diameter, $c_{P}$ is the speed of sound and $N$ is a characteristic number of acoustic transversals for a particle to adjust to its surrounding pressure (typically around 3-5). For small particles, values of $\tau$ are often fractions of nanoseconds - introducing numerical stiffness in the solution of the gas and solid phase equations. Approximations for reducing the difficulties associated with solving this term can be found in Ruggirello et al. (2010). For the present study, the work from compaction is solved using a pressure equilibrium procedure through a fractional time stepping approach. Details of this are given in Section 3.

Substituting Eqs. (13) and (14) into (11) results in the following set of final equations describing the compressible particulate phase:

$\dot{\alpha}_{s}=\left(\boldsymbol{D}_{s} / \rho_{s}\right) \cdot \nabla \alpha_{s}+\rho n_{P}\left(V_{P}^{\ddagger}+c_{P}^{\ddagger} / \gamma_{P}\right)$

$\dot{Y}_{s}=\left(\nabla \cdot \boldsymbol{D}_{s}\right) / \rho+n_{P} C_{P}^{\ddagger}$

$\dot{Y}_{s, i}=\left(\nabla \cdot \boldsymbol{D}_{s}\right) / \rho+\alpha_{s} \dot{m}_{s, i}^{\prime \prime \prime} / \rho+n_{P} C_{P, i}^{\ddagger}$

$\dot{\boldsymbol{u}}_{s}=\left(\boldsymbol{D}_{s} \cdot \nabla \boldsymbol{u}_{s}\right) /\left(\rho Y_{s}\right)-\alpha_{s} \nabla p_{s} /\left(\rho Y_{s}\right)+\left(n_{P} / Y_{s}\right) \int \underset{\sim_{P}}{\boldsymbol{\sigma}^{\prime}} \cdot \boldsymbol{n}_{p} d A_{P}$

$$
\begin{aligned}
\dot{e}_{s}= & \left(\boldsymbol{D}_{s} \cdot \nabla e_{s}\right) /\left(\rho Y_{s}\right)-\alpha_{s} p_{s} \nabla \cdot \boldsymbol{u}_{s} /\left(\rho Y_{s}\right) \\
& -\left(n_{P} / Y_{s}\right)\left[\int \dot{q}_{P}^{\prime \prime} d A_{P}+\left(p_{P}-\beta_{P}\right) V_{P}^{\ddagger}\right]
\end{aligned}
$$

where the heating rate of the particle and stress force along the particle surface can be related to the gas phase solution through interface matching conditions across the particle - gas interface.

In summary, the collections of equations in (5) and (11) are sufficient to describe a two-phase system consisting of a mixture of gases and particles given that particle level closures are provided for the phase coupling terms. These closures, in general, require knowledge of the micro-mechanics of the particulate field which can be postulated based on thermodynamic principals (Truesdell, 1984; Drew and Passman, 1999), asymptotic theories (Torquato, 2002) or localized solution approaches (Drew and Passman, 1999). In the present effort, a local solution approach is used based on an extension of the particle model of DesJardin et al. (2005) and Ruggirello et al. (2010). The appeal of the following local solution approach is that it accounts for solid phase compressibility effects at the particle level and is therefore compatible with the previously derived equations. The details of this model and its use in a reaction progress variable description is discussed next.

\subsection{Aluminum particle combustion model}

The aluminum particle model is divided into two stages of ignition and quasi-steady burning illustrated in Fig. 2. The ignition of aluminum particles begins with a relatively short heterogeneous surface reaction (HSR) stage (Fig. 2a) and quickly transitions to a quasi-steady diffusion flame (Fig. 2b) with a detached spherical flame positioned off the particle surface at two to five radii (Bucher et al., 1996; Dreizin, 1996). During the ignition phase, the particle heats up from convection and volumetric compression processes to its melting temperature. Fig. 2a shows the relatively short lived HSR stage consisting of solid metal $\left(m_{s}\right)$ and liquid metal $\left(m_{l}\right)$ surrounded by a layer of metal oxide $\left(m_{\operatorname{mox}-c}\right)$. Upon heating from convection and volumetric compression, the particle temperature increases until a melting phase transition occurs. If $m_{l}>0$ then metal oxide is allowed to form at the liquid/metal oxide interface as a result of oxygen having diffused through the metal oxide shell along its grain boundaries. Other diffusion mechanisms may also be present but these mechanisms are thought to be of secondary importance in comparison to grain boundary diffusion and therefore are neglected (Atkinson, 1985; Schtze, 2008).

At sufficiently high temperature and mechanical loading, the particle oxide layer peels back and collects as a cap allowing for molten aluminum to evaporate and form a diffusion flame (Rosenband, 2004). At high enough pressure it is possible that the aluminum particle will become supercritical and lack a well defined liquid/vapor phase boundary, but these effects were not considered in the present model. A review of supercritical (and subcritical) behavior and modeling is provided by Bellan (2005). As the particle burns, solid oxide condensates formed in the diffusion flame diffuse outward (denoted as $\operatorname{mox}-\infty$ ) and also towards the particle surface (denoted as $\operatorname{mox}-c$ ). The solid condensate that reaches the particle surface is assumed to accumulate on the leeward side of the particle, forming a cap. This cap serves to reduce the overall surface area of the particle, and in some cases, has been observed to result in violent surface gas ejection due to dilution of molten aluminum with oxides along with the participation of nitride reactions (Dreizin, 1999, 2000; Bucher et al., 1999). The vapor phase combustion is treated using an extension of the conserved scalar formulations for hydrocarbon droplets (Glassman, 1996). In this approach, standard approximations are employed that readily allow for a semi-analytical solution to the gas phase system. These approximations include a unity Lewis number, $\gamma_{P} D_{m}$ is a constant (i.e., Chapman gas assumption) and constant specific heats. Therefore the steady-state, 1D spherical, transport equations are the same as classical hydrocarbon droplet analysis except that the total mass flux is interpreted to be the sum of fluxes due to gas plus metal oxide. The exact phase of the metal oxide (i.e., solid vs. gas) is not delineated since it is assumed that the diffusion of the

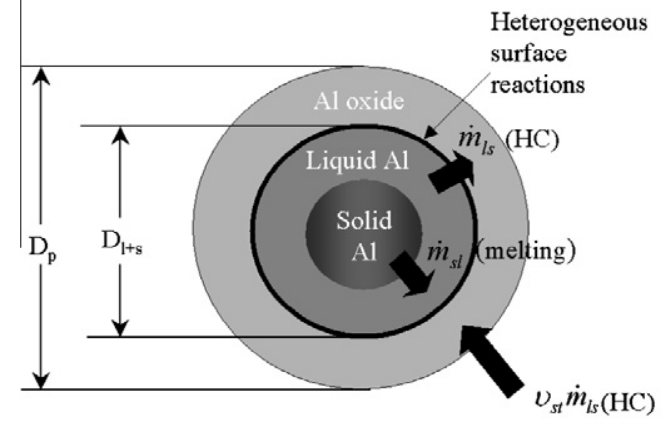

(a)

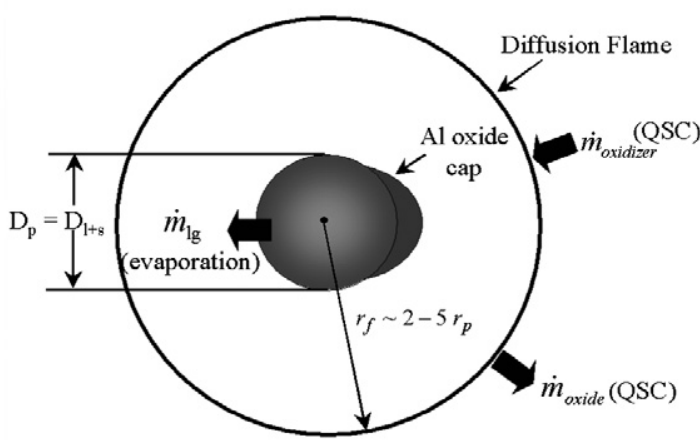

(b)

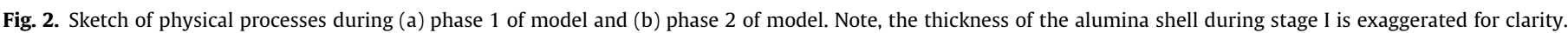


small metal oxide particles in its fume is the same as that of the gaseous species. This appears to be reasonable since the products of combustion include gaseous sub-oxide species such as $\mathrm{AlO}, \mathrm{AlO}_{2}$ and $\mathrm{Al}_{2} \mathrm{O}$ which are thought to condense out while forming $\mathrm{Al}_{2} \mathrm{O}_{3}$ as they diffuse from the flame to the particle surface. In addition, effects of thermophoretic diffusion also push the metal oxide particles from the hot flame to the relatively cooler surface. The current approximations may therefore account for the leading order effect of thermophoretic diffusion since the temperature and species gradients are proportional to each other. The novelty of the analysis is the inclusion of oxide deposition $\left(\dot{S}_{\text {mox }}^{\prime \prime}\right)$ on the particle surface through the surface boundary conditions to account for the deposition of metal oxide, resulting in a modification of the usual ShvabZel'dovich coupling functions (DesJardin et al., 2005).

The local particle solution is described with lumped approximations to describe the phase changes of the aluminum and its cap formation as a collection of ordinary differential equations that is expressed in terms of the grouping of the phase-coupling source terms that appear in Eq. (11) and also in terms of (unknown) surface quantities on the particle surface (to be defined),

$\frac{d m_{l}}{d t}=c_{P, l}^{\ddagger}= \begin{cases}\dot{m}_{s l}-\dot{m}_{l s} & \text { stage I } \\ \int \dot{m}_{l}^{\prime \prime} d A_{P} & \text { stage II }\end{cases}$

$\frac{d m_{m o x-c}}{d t}=c_{P, \text { mox }-c}^{\ddagger}= \begin{cases}v_{o x} \dot{m}_{l s} & \text { stage I } \\ -\int \dot{S}_{\text {mox }}^{\prime \prime} d A_{P} & \text { stage II }\end{cases}$

$\frac{d m_{\text {mox }-\infty}}{d t}=c_{P, \text { mox }-\infty}^{\ddagger}= \begin{cases}0 & \text { stage I } \\ \int\left[\left(1+v_{o x}\right) \dot{m}_{l}^{\prime \prime}-\dot{S}_{\text {mox }}^{\prime \prime}\right] d A_{P} & \text { stage II }\end{cases}$

$m_{P} \frac{d \boldsymbol{u}_{P}}{d t}=\int \underset{\sim P}{\boldsymbol{\sigma}^{\prime}} \cdot \boldsymbol{n}_{P} d A_{P} \quad$ stages I \& II

$m_{P} \frac{d e_{P}}{d t}=-\int \dot{q}_{P}^{\prime \prime} d A_{P}-\left(p_{P}-\beta_{P}\right) V_{P}^{\ddagger} \quad$ stages I \& II

where the subscripts $l, s$ and $P$ denote properties of the liquid, solid and average metal particle properties, respectively, $v_{o x}(=0.886)$ is the stoichiometric amount of oxygen consumed per unit mass of metal. In Eq. (16e) $e_{P}$ refers to the total mass weighted energy of both the aluminum and alumina (i.e., $e_{P}=\left(Y_{s+l} e_{\mathrm{Al}}+\left(Y_{m o x-c}\right.\right.$ $\left.\left.\left.+Y_{\text {mox }-\infty}\right) e_{\text {mox }}\right) /\left(Y_{s+l}+Y_{\text {mox }-c}+Y_{\text {mox }-\infty}\right)\right)$. A tabular SESAME (Kerley, $1991)$ EOS is used for both the solid constituents which is valid over the solid and liquid phases and includes the heats of melting and formation where appropriate. Therefore no terms are necessary in Eq. (16e) to account for the heat of melting for aluminum or the heat of formation for aluminum oxide. Eq. (16a) describes the time rate of change of the liquid mass for the droplet. Since the heat of melting for aluminum is already included in the EOS, Eq. (16a) is superfluous but it is included because it is used in the reaction progress variable formulation, described in Section 2.3, to track the melting of the particles. These equations have source and sink terms associated with aluminum melting $\left(\dot{m}_{s l}\right)$ and $\operatorname{HSR}\left(\dot{m}_{l s}\right)$, respectively (see Fig. 2a) and are determined from the following relations:

$$
\dot{m}_{s l}= \begin{cases}\left(-\int \dot{q}_{P}^{\prime \prime} d A_{P}-\dot{m}_{s l} h_{r, s l}\right) / h_{l s} & \text { for } 0 \leqslant \frac{m_{l}}{m_{s}}<1 \\ 0 & \text { and } e_{P}=e_{M, A l} \\ 0 & \text { otherwise }\end{cases}
$$

$\dot{m}_{l s}= \begin{cases}A_{s l s} A_{1} \exp \left(-E_{A} / R T_{\text {sls }}\right) & \text { for } 0 \leqslant \frac{m_{l}}{m_{s}}<1 \\ 0 & \text { otherwise }\end{cases}$ where $h_{l s}=396 \mathrm{~kJ} / \mathrm{kg}$ is the heat of melting and $h_{r, l s}=-31,000 \mathrm{~kJ} / \mathrm{kg}$ is the heat of reaction for the liquid surface reactions. The quantity $e_{M, A l}$ in Eq. (17) is the energy at melting which is defined from the tabular SESAME EOS for aluminum as the energy at the melting temperature of aluminum and $1 \mathrm{~atm}$ of pressure. This criteria for melting is chosen because while the melting temperature is a function of pressure, the energy at melting is found to be relatively constant by examining the phase boundary in the SESAME EOS. The HSR kinetic parameters $A_{1}=200 \mathrm{~kg} / \mathrm{m}^{2}$ and $E_{A}=95,395 \mathrm{~J} / \mathrm{mol}$ are taken as constants from the experimental study of Roberts et al. on the shock ignition of aluminum particles (Roberts et al., 1993), but in general are functions of the surrounding gas pressure and oxidizing environments. These sensitivities, however, are not accounted for in the present model. The material consumed from HSR is assumed to be small so that the surface area of the heterogeneous reaction front, $A_{s l s}$, is set equal to the surface area of the particle, $A_{P}$. The transition from stage I ignition to stage II combustion is assumed to be a function of a transition temperature that is chosen arbitrarily to be $T_{\text {trans }}=2000 \mathrm{~K}$. The model has been shown to be relatively insensitive to the exact value of the transition temperature (DesJardin et al., 2005).

The integral terms in Eqs. (16a)-(16e) represent mass, momentum and energy fluxes across the control volume interface. In Eq. (16d) the integral term involving the Cauchy stress tensor is simplified using a momentum balance relation across the gas-particle interface,

$-\underset{\sim}{\boldsymbol{\sigma}_{l}^{\prime}} \cdot \boldsymbol{n}_{P}=p_{g} \boldsymbol{n}_{P}+\boldsymbol{F}_{D}^{\prime \prime} \quad$ stage I

$-\underset{\sim_{l}}{\boldsymbol{\sigma}^{\prime}} \cdot \boldsymbol{n}_{P}=\left(\dot{m}_{l}^{\prime \prime}-\dot{S}_{\operatorname{mox}}^{\prime \prime}\right)\left(\boldsymbol{u}_{g}-\boldsymbol{u}_{l}\right)+p_{g} \boldsymbol{n}_{P}+\boldsymbol{F}_{D}^{\prime \prime} \quad$ stage II

where $\boldsymbol{F}_{D}^{\prime \prime}\left(=\boldsymbol{F}_{D} / A_{P}\right)$ is the average particle drag force (viscous and pressure) per unit area and $p_{g}$ is the mean gas pressure around the particle. Substitution of Eq. (19) into Eq. (16d) and assuming that the mass flux is evenly distributed over the surface (i.e., effects of particle jetting and spinning are neglected) then the following result is obtained:

$m_{P} \frac{d \boldsymbol{u}_{P}}{d t}=\frac{\pi}{8} \rho_{g} D_{P}^{2} C_{D}\left|\boldsymbol{u}_{g}-\boldsymbol{u}_{P}\right|\left(\boldsymbol{u}_{g}-\boldsymbol{u}_{P}\right)$

where the drag force is approximated as: $\boldsymbol{F}_{D}=(\pi / 8)$ $\rho_{g} D_{P}^{2} C_{D}\left|\boldsymbol{u}_{g}-\boldsymbol{u}_{P}\right|\left(\boldsymbol{u}_{g}-\boldsymbol{u}_{P}\right)$ and $C_{D}$ is the drag coefficient expressed in terms of a particle Reynolds number, $\operatorname{Re}_{P}\left(=D_{P} \rho_{g}\left|\boldsymbol{u}_{P}-\boldsymbol{u}_{\mathrm{g}}\right| / \mu_{\mathrm{g}}\right)$, using standard drag laws for flow over a sphere.

$C_{D}= \begin{cases}24\left(1+\operatorname{Re}_{P}^{2 / 3} / 6\right) / \operatorname{Re}_{P} & \text { for } \operatorname{Re}_{P} \leqslant 1000 \\ 0.424 & \text { for } \operatorname{Re}_{P}>1000\end{cases}$

While more refined models for particle drag in high Mach number flow are available (Smirnov et al., 1996; Zhang et al., 2003; Ling et al., 2011a,b) the more simplified approach here has been shown to be adequate for application to detonation environments (Papalexandrix, 2005).

For stage I, a balance of energy across the solid-gas interface requires $\dot{q}_{P}^{\prime \prime}=\dot{q}_{g}^{\prime \prime}+\dot{q}_{r a d}^{\prime \prime}$, where $\dot{q}_{g}^{\prime \prime}=-k_{g} \nabla T \cdot \boldsymbol{n}_{P}$ and $\dot{q}_{r a d}^{\prime \prime}$ is the net radiation flux leaving the particle surface. A Nusselt number correlation is used to model the particle heat transfer, $\int \dot{q}_{g}^{\prime \prime} d A_{P}=$ $\pi D_{P} \mu_{g} C_{P, g} / \operatorname{Pr}_{g}\left(T_{g}-T_{P}\right) N u_{P}^{I}$, where $N u_{P}^{I}=2\left[1+\operatorname{Re}_{P}^{1 / 2} \operatorname{Pr}_{g}^{1 / 3} / 3\right]$ and $P r_{g}$ is set equal to 0.613 . While more refined models for heat transfer in shocked environments exist (Smirnov et al., 1996), they are not explored in the present study.

For stage II, $\dot{q}_{P}^{\prime \prime}$ is defined by the heat flux from the flame and evaporation processes at the particle surface. A local gas-phase solution to the coupled heat and mass transfer problem is based 
on the use of Shvab-Zel'dovich coupling functions that are defined by DesJardin et al. (2005),

$B_{m-o x}=\frac{Y_{m, s l g}+Y_{o x, \infty} / v_{o x}}{f+1-Y_{m, s l g}}$

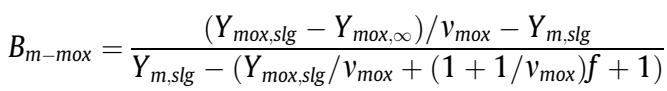

$B_{m-p}=\frac{\left(Y_{p, s l g}-Y_{p, \infty}\right) / v_{p}-Y_{m, s l g}}{Y_{m, s l g}-\left(Y_{p, s l g} / v_{p}+f+1\right)}$

$B_{I}=\left(Y_{I, \infty} / Y_{I, s l g}\right)-1$

$B_{m-T}=\frac{C_{P, s l g}\left(T_{\infty}-T_{s l g}\right)+Y_{m, s l g} h_{r, g s}}{h_{l g}^{\prime}+h_{r, g s}\left(f+1-Y_{m, s l g}\right)}$

$f=-\frac{\left(1-Y_{m, s l g}\right)\left(Y_{m o x, \infty} / v_{m o x}-Y_{o x, \infty} / v_{o x}\right)}{\left(Y_{m o x, \infty}-Y_{m, s l g}\right) / v_{m o x}-\left(1+1 / v_{m o x}\right) Y_{o x, \infty} / v_{o x}}$

where the subscript slg denotes a gas property at the surface of the liquid-gas interface, $\operatorname{mox}$ is a metal oxide property, $\infty$ is a far field property (set equal to the mass fractions for air), $I$ is an inert (taken as $\mathrm{N}_{2}$ ), ox is the oxidizer (taken as $\mathrm{O}_{2}$ ) and $p$ are the product gases. The quantity $f \equiv \dot{S}_{\operatorname{mox}}^{\prime \prime} / \dot{m}_{T}^{\prime \prime}$, is defined as the ratio of the rate of metaloxide deposited into the oxide cap $\left(\dot{S}_{\operatorname{mox}}^{\prime \prime}\right)$ to the net gas mass flux at the surface $\left(\dot{m}_{T}^{\prime \prime}=\dot{m}_{l}^{\prime \prime}-\dot{S}_{\text {mox }}^{\prime \prime}\right)$ and solved as part of the eigenvalue problem. The quantities $h_{r, g s}(=-43,334.82 \mathrm{~kJ} / \mathrm{kg})$ is the vapor phase heat of reaction and $h_{l g}^{\prime}$ is the effective latent heat of vaporization defined by,

$h_{l g}^{\prime}=h_{l g}(f+1)-\Delta K E+\frac{\dot{q}_{r a d}^{\prime \prime}-\dot{q}_{P}^{\prime \prime}}{\dot{m}_{T}^{\prime \prime}}$

where $h_{\text {lg }}(=11,834.82 \mathrm{~kJ} / \mathrm{kg})$ is the latent heat of evaporation. The change in kinetic energy $\left(\Delta K E=\left|\boldsymbol{u}_{P}-\boldsymbol{u}_{g}\right|^{2} / 2\right)$ in Eq. (23) is a newly introduced term that arises from energy balance considerations across the liquid-gas interface. Eqs. (22a)-(22f) along with a vapor pressure relation for aluminum (Hultgren et al., 1973),

$p_{\mathrm{Al}, \text { vap }}=\exp \left[36.547-\frac{39,033}{T_{\text {slg }}}-1.3981 \ln T_{\text {slg }}+6.7839 \cdot 10^{-9} T_{\text {slg }}^{2}\right]$

represent a system of non-linear algebraic equations to determine the mass and heat fluxes along with the thermodynamic state at the surface. Details on the numerical solution approach for solving these equations is summarized in DesJardin et al. (2005). The result provides a solution to the mass flux terms required in Eq. (16a) and heat flux in Eq. (16e) for the stage II of the particle resulting in the following final form of the particle equations:

$$
\begin{aligned}
& \frac{d m_{l}}{d t}=c_{P, l}^{\ddagger}= \begin{cases}\dot{m}_{s l}-\dot{m}_{l s} & \text { stage I } \\
(f+1) \dot{m}_{P} & \text { stage II }\end{cases} \\
& \frac{d m_{m o x-c}}{d t}=c_{P, \text { mox }-c}^{\ddagger}= \begin{cases}v_{o x} \dot{m}_{l s} & \text { stage I } \\
f \pi D_{P} \frac{\mu_{g}}{S c_{g}} B S h_{g} & \text { stage II }\end{cases} \\
& \frac{d m_{m o x-\infty}}{d t}=c_{P, \text { mox }-\infty}^{\ddagger}= \begin{cases}0 & \text { stage I } \\
\left.\left(1+v_{o x}\right)(1+f)\right) & \text { stage II } \\
\pi D_{l} \frac{\mu_{g}}{S c_{g}} B S h_{g} & \text { sta }\end{cases}
\end{aligned}
$$

$m_{P} \frac{d \boldsymbol{u}_{P}}{d t}=\frac{\pi}{8} \rho_{g} D_{P}^{2} C_{D}\left|\boldsymbol{u}_{g}-\boldsymbol{u}_{P}\right|\left(\boldsymbol{u}_{g}-\boldsymbol{u}_{P}\right)$

stages I \& II

$$
m_{P} \frac{d e_{P}}{d t}= \begin{cases}\pi D_{P} \frac{\mu_{g} C_{P, g}}{P r_{g}}\left(T_{g}-T_{P}\right) N u_{P}^{I}-\dot{q}_{r a d}^{\prime \prime} A_{P} & \\ -\left(p_{P}-\beta_{P}\right) V_{P}^{\ddagger} & \text { stage I } \\ \pi D_{P} \frac{\mu_{g} C_{P, g}}{P r_{g}} \Delta T N u_{P}^{I I}-\dot{q}_{r a d}^{\prime \prime} A_{P} & \\ -\left(p_{P}-\beta_{P}\right) V_{P}^{\ddagger} & \text { stage II }\end{cases}
$$

The quantity $D_{P}$ is the effective total particle diameter defined as $D_{P}=\left[6\left(m_{P} / \gamma_{P}\right) / \pi\right]^{1 / 3}$. The conservation equations are expressed in terms of effective Nusselt $\left(N u_{P}\right)$ and Sherwood $\left(S h_{P}\right)$ numbers so simple corrections can be easily included to account for convective flow effects. For stage II, vapor phase burning, the Ranz-Marshall correlations (Ranz and Marshall, 1952; Sirignano, 1999) are used i.e., $N u_{P}^{I I}\left(S h_{P}\right)=2\left[1+\operatorname{Re}_{P}^{1 / 2}\left[\operatorname{Pr}_{g}\left(S c_{g}\right)\right]^{1 / 3} / 3\right] \ln (1+B) / B$, where $S c_{g}=$ $\mathrm{Pr}_{\mathrm{g}}=0.613$. The effective temperature difference $\Delta T$ given in Eq. (25f) that is compatible with the local eigenvalue gas phase analysis is given as: $\Delta T=T_{\infty}-T_{s l g}-h_{r, g s}\left[B(f+1)-Y_{m, s l g}(1+B)\right] / C_{P, g}$, where $T_{\text {slg }}$ is the temperature at the surface of the liquid-gas interface and $h_{r, g s}$ is the heat of reaction associated with aluminum vapor forming solid aluminum oxide particles in the diffusion flame. The quantity $\dot{q}_{\text {rad }}^{\prime \prime}$ is the net radiation heat flux from the surface of the particle from thermal radiation. The contribution of this term to the energy balance is neglected for all cases considered in this study.

\subsection{A reaction progress variable description of aluminum combustion}

While the use of the Lagrangian description of the aluminum particle burn model of Section 2.2 has been used directly in coupled simulations of multiphase flows (Ruggirello et al., 2010), an Eulerian based reaction progress variable description of the burning particulate is desirable in the present study for three main reasons. The first is the limitations of the CTH shock physics code which currently does not support Lagrangian-Eulerian descriptions, therefore an Eulerian description is a necessity. The second is the desire to account for group combustion modes burning (Chiu and Liu, 1977) for aluminum rich explosives that requires knowledge of the local environment. The third is the ability of the formulation to readily account for the effects of unresolved sub-grid scale (SGS) turbulence via single point joint probability density function (PDF) descriptions. The appeal of reaction progress variable descriptions in this context is to reduce the degrees of freedom describing the reaction processes thereby simplifying the treatment of the joint PDF describing the composition state, although the effects of SGS mixing are not considered in the present study.

To reduce the number of degrees of freedom, it is desirable to define the composition state, specified by mass fractions $\left(Y_{i}\right)$, in terms of mixture fraction $(Z)$ and reaction progress variables $\left(C^{\prime} s\right)$ defined by the functional relation, $Y_{i}(\boldsymbol{x}, t)=Y_{i}\left(Z(\boldsymbol{x}, t), C_{k}(\boldsymbol{x}, t)\right)$, where the subscript $i$ denotes one of the constituents. The state relations may be specified from detailed experimental measurements (Bilger, 1977), direct numerical simulations, reduced modeling descriptions (e.g., flamelet descriptions (DesJardin, 2005)) or through conditional moment equations which, in turn, require further modeling assumptions (Bilger, 1993). Previous studies using reaction progress variables include LES of ignition and extinction processes (Pierce and Moin, 2004; Ihme and Pitsch, 2008), formation of soot (Kumar and DesJardin, 2007), and partially premixed flames (Bilger, 2000). An excellent introduction to the use of reaction progress variable descriptions can be found in the text by Fox (2003).

In this study, a reaction progress variable description for the two-phase system is formulated using the mixture fraction $(Z)$ and four reaction progress variables describing the particle processes of melting, heterogeneous surface reactions (HSR), quasisteady burning (QSB) and group combustion (GC). The precise 
definition of $Z$ is critical in defining realizable limits of the statespace for the species mass fractions. Recently, Baba and Kurose (2008) and Reveilon and Vervisch (2005) conducted 2D DNS of spray flames and explored the flame structure in mixture fraction space for laminar and transitionally turbulent jet flames. In both studies, the definition of mixture fraction used was defined only in terms of gas phase of the system, i.e., $Z_{g}=m_{Z} / m_{g}$, where $m_{Z}$ is locally the amount of mass in the gas phase that originated from the liquid fuel stream and $m_{g}$ the local gas mass. Using this definition, the span of mixture fraction in the solution space will increase with evaporation, i.e., $Z_{g}=0$ at the onset of evaporation and $Z_{g} \rightarrow 1$ when all the fuel is evaporated. For the limiting case of a non-reacting flow (pure mixing), a unique mapping relationship between mixture fraction and the gaseous fuel can be expected since both are governed by nearly the same PDE (they differ by a scaling constant) and have similar boundary conditions. The same, however, cannot be said for the rest of the species in the system since there is no evaporation source/sink evaporation term associated with those species. A secondary progress variable describing evaporation would therefore have to be introduced to establish a unique state-map for the remaining species. For reacting sprays and particulate, the situation is further complicated requiring several progress variables to, not only account for the reactions, but also changes in the span of the mixture fraction space from evaporation. A mixture fraction based formulation based solely on either the gas or particulate phases of the system therefore appears to be of limited value.

Alternatively, if the mixture fraction is defined as the sum of the contributions from both phases, i.e., $Z \equiv \alpha_{g} Z_{g}+\alpha_{p} Z_{p}$ then a unique mapping for all species to mixture fraction can be established in the pure mixing limit if $\boldsymbol{D}_{i}=\boldsymbol{D}_{Z}$ is assumed for all species. In the limit of zero slip (i.e., a homogeneous flow model) then $\boldsymbol{D}_{i}=\boldsymbol{D}_{Z} \equiv 0$ and the mapping precisely defined. In this study, the mixture fraction is defined as locally the amount of mass that originated from the explosive that includes all phases of the post-detonated products (solid, gas and liquids). A multiphase definition of $Z$ is advantageous since it preserves scalar conservation - consistent with recent mixture fraction formulations of Bilger (2011) for spray flames. The transport equation for the mixture fraction is,

$\rho \dot{Z}=\nabla \cdot\left(Z \boldsymbol{D}_{Z}\right)$

where no source or sink appear in Eq. (26) since the sum of the homogeneous and heterogeneous reaction source terms are identically equal to zero. Furthermore, an exact state relation can be established for all species in the limits of either pure mixing or infinitely fast chemistry. The latter fact is useful for defining a group combustion progress variable to be discussed in Section 2.3.5.

The species constituents considered are solid metal $(s)$, liquid metal $(l)$, metal vapor $(v)$, oxidizer $(o x)$, inert species $(I)$, and alumina that either collects in the cap of the particle $(\operatorname{mox}-c)$ or is dispersed to the far-field as fine particulate $(\operatorname{mox}-\infty)$. A canonical problem to deduce state relations for highly compressible shock driven reactive mixtures is nontrivial. Since special care is taken to define the mixture fraction, two limits of the state space are examined and provide guidance for constructing the reaction progress variables. The first is the limit of pure mixing of an evaporating dispersed cloud of $\mathrm{Al}$ particles. In this limit, pure mixing state relations are expected between the mass fractions of all species $Y_{i}$ and $Z$ and illustrated by the solid lines in Figs. 3a, 4a and 5a. The second limit is the case when all of the available oxygen is consumed in the cloud of particulate resulting in an aluminum rich pocket of vapor that burns in a diffusion flame. This limit is illustrated by the dashed lines in Fig. 6a showing the effects of the group combustion progress variable on $Y_{i}$, eventually resulting in a high group combustion number $(G)$ limit. It should be emphasized that all of the state relationships span the entire range of mixture fraction limits from 0 to 1 - analogous to state maps defined for single gas phase flows.

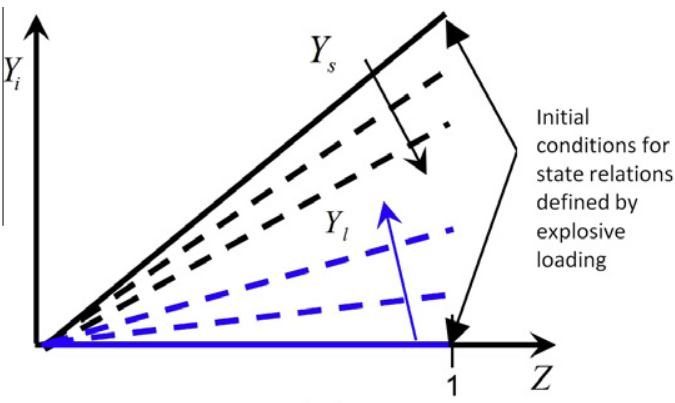

(a)

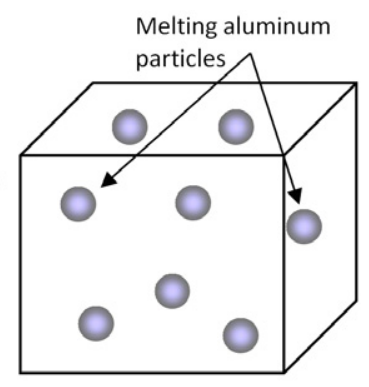

(b)

Fig. 3. Sketches of (a) changes in state relations with increasing $C_{M}$ and (b) physical interpretation of the processes.

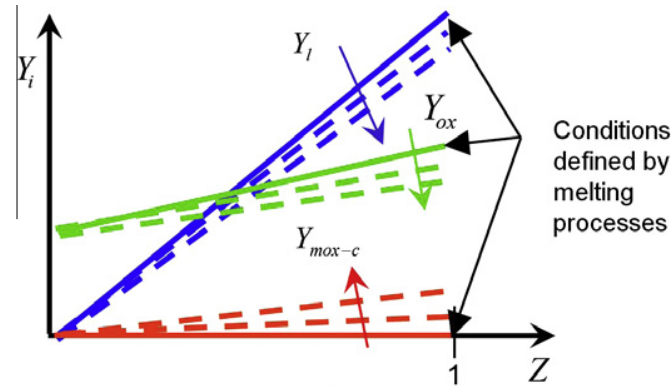

(a)

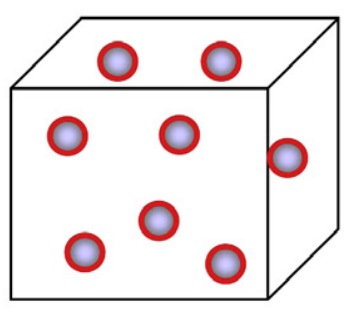

(b)

Fig. 4. Sketches of (a) changes in state relations with increasing $C_{H S R}$ and (b) physical interpretation of HSR reactions. 


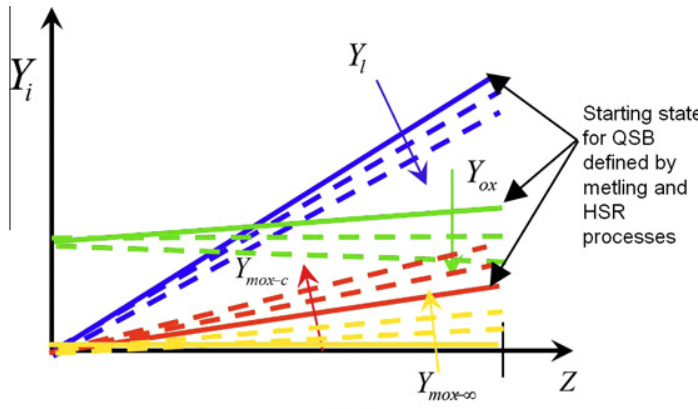

(a)

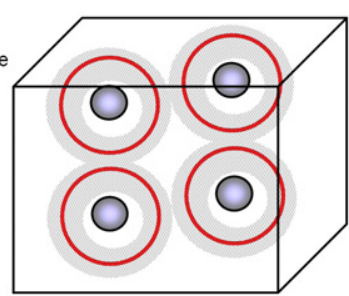

(b)

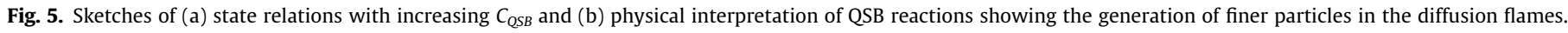

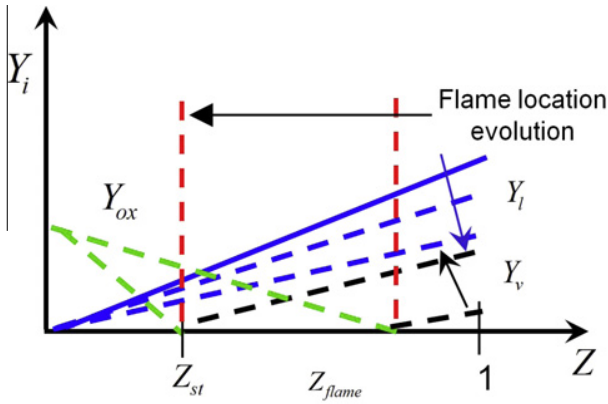

(a)

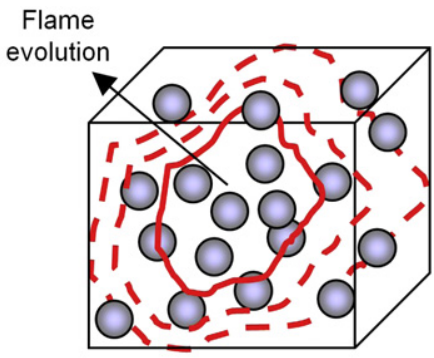

(b)

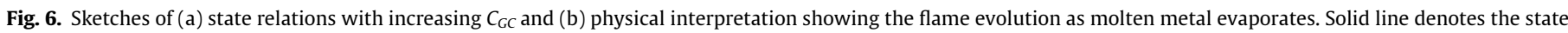
after QSB.

Describing the evolution of the state relations from the pure mixing to the high $G$ limit requires additional progress variables describing melting $\left(C_{m}\right)$, HSR $\left(C_{H S R}\right)$, QSB $\left(C_{Q S B}\right)$ and GC $\left(C_{G C}\right)$. Ideally, DNS could be conducted to extract the dependence of $Y_{i}$ and also the dependence of $C_{m}, C_{H S R}, C_{Q S B}$ on $Z$, however, a simple conical problem for shock driven explosives is difficult to define and nearly impossible to conduct using DNS. Alternatively, an assumed functional dependence is pursued based on some physical reasoning for each of these process. The rate of melting, HSR and QSB will be independent of the value of $Z$ since those processes are particle dependent. A linear dependence of $Y_{i}\left(Z, C_{k}\right)$ on $Z$ therefore appears appropriate, as illustrated in Figs. 3a, 4a and 5a by the linear solid and dashed lines. The role of the progress variables for these stages is to effectively change the slope of the state relations. The transition to QSB to GC for $Y_{v}, Y_{o x}$ and $Y_{m o x-\infty}$ from the pure mixing to infinitely fast chemistry state relations is less certain. This transition is illustrated in Fig. 6 showing the transition from QSB to a high GC limit. As will be described in Section 2.3.5, while the exact mass fraction transition dependence is phenomenologically based, the rate of transition is dependent on droplet evaporation that is consistent with group combustion concepts, i.e., the transition from low to high $G$ limits.

Additional details of the state relations and definition of the progress variables are described in the following sections which define four distinct particle processes of melting, HSR, QSB and GC. The transition from one state to the next is driven by the source terms for the reaction progress variables that are defined using the aluminum particle model description of Section 2.2.

\subsubsection{State of post-detonated explosive}

The initial state of the post-detonated products follows a pure mixing state relationship, $Y_{i}^{\text {ref }}(Z)=Y_{i}^{\infty}+\left(Y_{i}^{o}-Y_{i}^{\infty}\right) Z$, where $Y_{i}^{\infty}$ and $Y_{i}^{o}$ are the far-field and explosive species mass fractions, respectively, and are shown as solid lines in Fig. 3. Oxidizer is assumed to be present in both the detonation products as well as in the far-field for most cases. The values of $Y_{i}^{\infty}$ are assumed to be that of the gas surrounding the charge. The values of $Y_{i}^{o}$ are determined using two parameters describing the overall equivalence ratio of the post-detonated mixture, $\Phi=v_{o x} Y_{\mathrm{Al}}^{o} / Y_{o x}^{o}$, and an oxygen factor defined as $F=Y_{o x}^{o} /\left(Y_{o x}^{o}+Y_{I}^{o}\right)$. The limits of $F=0$ and 1 correspond to the limiting cases where there is no oxidizer and all oxidizer in the post-detonated products. With these two parameters, the initial composition field consisting of solid metal $(s)$, oxidizer (ox) and inert $(I)$ can be determined using the following relations:

$Y_{s}^{o}=\frac{\Phi F}{\Phi F+v_{o x}}, \quad Y_{o x}^{o}=\frac{F v_{o x}}{\Phi F+v_{o x}}, \quad Y_{I}^{o}=\frac{v_{o x}(1-F)}{\Phi F+v_{o x}}$

From this prescribed post-detonation state, the metal particles will undergo melting, HSR, QSB and possibly GC if in a fuel rich environment.

\subsubsection{Melting progress variable $\left(C_{M}\right)$}

During the melting stage of the aluminum, the initial solid phase of the aluminum is converted into liquid metal and can be defined by the following reaction progress variable:

$C_{M}=\frac{Y_{l}(\boldsymbol{x}, t)}{Y_{s}^{\text {ref }}(Z)}$

where $Y_{s}^{\text {ref }}$ is defined as the (known) mass fraction of the solid aluminum from the post-detonation products. A progress variable to track the melting of the particles is needed in order to determine if QSB can happen. If $C_{M}<1$ the particles are only partially melted and the particles cannot transition to QSB until they are fully 
melted. Using Eq. (28) the transport for $C_{M}$ can be explicitly derived from Eq. (15c) assuming the drift term for $Z$ is the same at that for the particles (i.e. $\boldsymbol{D}_{Z}=\boldsymbol{D}_{P}$ ),

$\dot{C}_{M}=\frac{\nabla \cdot \boldsymbol{D}_{P}}{Y_{s}^{\text {ref }} \rho}+S_{C_{M}}$

where the source term, $S_{C_{M}}$, is a function of the particle number density, and the particle source term associated with melting given in Eq. (17), i.e. $S_{C_{M}}=n_{p} \dot{m}_{s l} / Y_{s}^{r e f}$.

The particle source term is a function of the gas and particle composition and temperatures. With the values of $C_{M}$ and $Z$, the composition field during melting is assumed to have a linear dependence on $Z$ and $C_{M}$,

$Y_{i}^{M}=Y_{i}^{r e f}(Z)+v_{i} C_{M} Y_{s}^{r e f}(Z)$

where $v_{i}$ is the mass of the $i$ th species consumed or produced during the melting process per unit mass of solid aluminum melted, i.e., $v_{l}=1, v_{s}=-1$ and $v_{i}=0$ for the remaining species. The resulting functional description of $Y_{l}$ and $Y_{S}$ on $C_{M}$ is illustrated in Fig. 3 showing (a) changes in the state relationships with increasing values of $C_{M}$ (dashed lines) and (b) a sketch of the physical interpretation of the SGS process during melting.

\subsubsection{HSR progress variable $\left(C_{H S R}\right)$}

After melting is initiated, HSR occurs and an associated reaction progress variable is defined in terms of the local oxygen mass fractions,

$C_{H S R}=\frac{Y_{o x}^{r e f}(Z)-Y_{o x}}{Y_{o x}^{r e f}(Z)}$

where $Y_{o x}^{\text {ref }}$ is defined from the post-detonation products assuming the pure mixing state relation. A similar transport equation to that of Eq. (29) can also be derived to describe its evolution and is tracked as part of the simulation with an associated source term, $S_{C_{H S R}}=n_{p} \dot{m}_{l s} v_{H S R, o x} / Y_{o x}^{r e f}$, where $\dot{m}_{l s}$ is the rate of liquid metal mass consumed given by Eq. (18). During HSR, the composition is assumed to follow a linear dependence in $Z$ illustrated in Fig. 4 (dashed lines) resulting in the following relation:

$Y_{i}^{H S R}\left(Z, C_{M}, C_{H S R}\right)=Y_{i}^{M}(Z)-v_{H S R, i} Y_{o x}^{r e f}(Z) C_{H S R}$

where $Y_{i}^{M}$ is determined from Eq. (30) and $v_{H S R, i}$ is the mass of the $i$ th species consumed or produced during the HSR. As shown, in the limit as $C_{H S R} \rightarrow 1$ then $Y_{o x} \rightarrow 0$ at the value of $Z=1$. In this limit, the resulting fuel rich mixture from the explosive could potentially exhaust the available local oxidizer.

\subsubsection{Quasi-steady burning progress variable $\left(C_{Q S B}\right)$}

Once the particle is sufficiently hot and fully melted from convective heat transfer and HSR, the alumina shell fractures and the particle transitions to a diffusion mode of burning with a flame located at 3-5 radii from the particle surface (Bucher et al., 1996; Dreizin, 1996). The associated reaction progress variable for this stage is defined as,

$C_{Q S B}=\frac{Y_{o x}^{H S R}(Z)-Y_{o x}}{Y_{o x}^{H S R}(Z)}$

where $Y_{o x}^{H S R}$ denotes the oxygen state at the end of the HSR stage for which the particles transition to QSB and therefore is only a function of $Z$ thereafter. Similar to the melting and HSR stages, $C_{Q S B}$ is assumed to follow a linear dependence on $Z$ :

$Y_{o x}^{Q S B}=Y_{o x}^{\infty}+\left[Y_{o x}^{o}\left(1-C_{H S R}\right)\left(1-C_{Q S B}\right)-Y_{o x}^{\infty}\right] Z$

resulting in the behavior of $Y_{o x}$ during the QSB stage as sketched in Fig. 5.
The left most boundary of the state map remains fixed while the right boundary changes due to oxidation. This will continue until the mass fraction of the oxidizer at $Z=1$ goes to zero corresponding to the condition, $C_{Q S B}=1$. After this point all the oxidizer present in the post-detonation products is completely consumed and any additional oxidation of the particles would come from mixing of the surrounding air. Using Eq. (33) an analytical expression for the remaining species can be developed.

$Y_{i}^{\mathrm{QSB}}=Y_{i}^{H S R}+v_{\mathrm{QSB}, i} Z\left(1-C_{H S R}\right) Y_{o x}^{o} / v_{\mathrm{QSB}, \mathrm{ox}}$

A transport equation for $C_{O S B}$ can be derived that is similar to Eq. (29) with an associated source term, $S_{C_{Q S B}}=n_{p} \dot{m}_{l} v_{Q S B, o x} /$ $\left(Y_{o x}^{o}\left(1-C_{H S R}\right) Z\right)$, where $\dot{m}_{l}$ is the rate of liquid metal mass consumed computed from Eqs. (25a) and (25c).

For fuel rich mixtures, if the local oxygen is completely consumed then $C_{Q S B} \rightarrow 1$. For fuel lean mixtures, the aluminum will be consumed with extra remaining oxygen. If the oxidation from surface reactions is small (typically the case) then $C_{H S R} \ll 1$ and $Y_{o x}^{H S R} \simeq Y_{o x}^{o}$. Under these conditions, if all of the aluminum burns then $C_{Q S B} \rightarrow\left(Y_{o x}^{o}-Y_{o x}\right) / Y_{o x}^{o}=\Delta Y_{o x} / Y_{o x}^{o}=\left(Y_{\mathrm{Al}, o} / Y_{o x, o}\right) / v_{o x}=\Phi$. As will be shown in the results, this limit is often achieved for the fuel lean explosives.

\subsubsection{Group combustion progress variable $\left(C_{G C}\right)$}

For fuel rich explosives where excess aluminum is present after QSB it is expected that the metal will burn in a group combustion mode (Chiu and Liu, 1977). In this case there is not sufficient penetration of the air into the droplet laden flow to sustain individual droplet burning. The rate of burning in this limit is therefore gas diffusion controlled vs. evaporation controlled. Group combustion concepts have been previously incorporated into modeling studies of spray flames and coal combustion but, to the authors' knowledge, it has not been used in the context of non-ideal explosives. Previous work by Chiu and Liu (1977) and Chiu et al. (1982) utilized a non-dimensional group combustion number $(G)$ representing the ratio of heat transfer in the gas phase to the heat transfer between the two phases. The $G$ parameter is used to differentiate between weak and strong droplet interactions. They identified four modes of group combustion. For low values of $G$ the droplets are far enough apart that isolated droplet combustion is dominant. For moderate values of $G$, the droplets become closer and the individual flames begin to combine so that the main flame is located somewhere within the cloud of droplets. Outside of this main flame isolated droplet burning is still observed. If $G$ is increased further the flame is pushed outside the group of droplets and a diffusion flame around the droplets is formed. In this case all the droplets are evaporating as a group and combustion takes place in the surrounding flame. Finally, for very large values of $G$ the droplets in the center of the group are saturated and do not evaporate. Only droplets located near the edge of the group evaporate and participate in the combustion. In this model both the internal and external group combustion modes, for moderate to high $G$ numbers, are resolved. Low values of $G$ for which individual droplet burning is dominant are already handled through the quasisteady burning mode discussed previously.

The initiation of group combustion is modeled using the criterion that $C_{Q S B}=1$ indicating the complete depletion of oxidizer in the richest regions of the flow. Physically it is expected that during this process a diffusion flame will be formed in the fuel rich regions of the flow, progressively moving outward toward the air until all of the remaining liquid metal from the QSB stage completely evaporates. This process is represented in state space in Fig. 6 showing (a) the changes in composition with increasing values of $C_{G C}$ and (b) a sketch of the expected flame behavior in physical space. 
The initiation of the flame location is assumed to travel from fuel rich to leaner regions with increasing values of $C_{G C}$ which is defined in terms of the amount of liquid metal,

$C_{G C}=1-\frac{Y_{l}}{Y_{l}^{Q S B}}$

where $Y_{l}^{Q S B}$ denotes the remaining amount of liquid metal after the QSB stage. The condition $C_{G C}=1$ denotes the limit when all of the remaining liquid metal has evaporated and the flame reaches the position in mixture fraction space, $Z_{s t}$, where the vapor metal and air are in stoichiometric proportions defined as,

$Z_{s t}=\frac{Y_{o x}^{o}}{Y_{o x}^{o}+v_{G C, o x} Y_{l}^{Q S B}}$

where $Y_{l}^{Q S B}$ denotes the mass fraction of liquid metal at $Z=1$ after QSB. The position of the diffusion flame from the fuel rich regions to its ultimate position at $Z_{s t}$ is assumed to be proportional to $C_{Q S B}$, i.e., $Z_{\text {flame }}=1-\left(1-Z_{s t}\right) C_{G c}$. Given $Z_{\text {flame }}$ then the aluminum vapor, oxidizer, and far-field metal oxide species mass fractions can be determined using the following relations assuming a thin diffusion flame:

$$
\begin{aligned}
& Y_{v}= \begin{cases}0 & \text { for } Z \leqslant Z_{\text {flame }} \\
Y_{l}^{Q S B} C_{G C}\left(Z-Z_{\text {flame }}\right) /\left(1-Z_{\text {flame }}\right) & \text { for } Z>Z_{\text {flame }}\end{cases} \\
& Y_{o x}= \begin{cases}Y_{o x}^{o}\left(Z_{\text {flame }}-Z\right) / Z_{\text {flame }} & \text { for } Z \leqslant Z_{\text {flame }} \\
0 & \text { for } Z>Z_{\text {flame }}\end{cases}
\end{aligned}
$$

$Y_{\text {mox }-\infty}= \begin{cases}\left.Y_{\text {mox }-\infty}\right|_{Z=Z_{\text {flame }}} Z / Z_{\text {flame }} & \text { for } Z \leqslant Z_{\text {flame }} \\ \left.Y_{\text {mox }-\infty}\right|_{Z=Z_{\text {flame }}}\left(Z-Z_{\text {flame }}\right) /\left(1-Z_{\text {flame }}\right) & \text { for } Z>Z_{\text {flame }}\end{cases}$

where $\left.Y_{\text {mox }-\infty}\right|_{Z=Z_{\text {flame }}}=\left(Y_{\text {mox }-\infty}^{Q S B}+C_{G C} v_{G C, \operatorname{mox}} Y_{l}^{Q S B}\right) Z_{\text {flame }}$. With the state relationships now defined, an evolution equation for $C_{Q S B}$ can be derived using the definition of Eq. (36) that is similar to that given in Eq. (29) and with an associated source term given as: $S_{C_{G C}}=n_{p} \dot{m}_{\text {evap }} / Y_{l}^{Q S B}$, where $\dot{m}_{\text {evap }}$ is the evaporation rate for an isolated liquid aluminum metal droplet (no burning). In this limit, $\dot{m}_{\text {evap }}$ is computed using the coupling functions $B_{m}=\left(Y_{m, s l g}-Y_{m, \infty}\right) /$ $\left(1-Y_{m, s l g}\right)$ and $B_{T}=C_{P, s l g}\left(T_{\infty}-T_{s l g}\right) / h_{l g}^{\prime}$.

\section{Numerical implementation}

The multiphase flow and reaction progress variable model are implemented into the CTH Shock Physics code (McGlaun et al., 1990). CTH is an Eulerian multi-material hydrodynamics code developed at Sandia National Laboratories for modeling complex large deformation and/or strong shock problems. A second order accurate solution algorithm is used to solve the conservation of mass, momentum, and energy equations in two steps, a Lagrangian step and a remap step. During the Lagrangian step the Lagrangian form of the conservation equations are integrated for a time step, during which the initial mesh distorts to follow the material motion. An explicit finite volume formulation is used to solve the momentum and energy conservation equations during the Lagrangian step, while mass is automatically conserved since there is no mass flux across the cell boundaries. After the Lagrangian step, the remap step remaps the distorted mesh back to the original fixed mesh. An interface tracking algorithm (Noh and Woodward, 1976; McGlaun et al., 1990) is used to decide which materials are moved with the volume flux during the remap step. A second order accurate monotonicity preserving conservative scheme developed by van Leer (1979) is used to perform the remap operation in each dimension separately using an operator splitting approach
To integrate the multiphase flow and reaction progress variable models into CTH a three stage fractional step method is used to integrate the system of PDEs due to the disparate time scales between convective processes and phase interactions. During the first fractional stage the total mixture equations, Eq. (5), solid phase transport equations, Eq. (11), mixture fraction, Eq. (26), and progress variable transport equations are advanced during the remap step. The drift terms and source/sink terms are neglected during this stage for the solid phase and progress variable transport equations resulting in the following set of conservation equations solved during this stage:

$$
\begin{aligned}
& \dot{\rho}=-\rho \nabla \cdot \boldsymbol{u} \\
& \rho \dot{u}=-\nabla p \\
& \rho \dot{e}=-p \nabla \cdot \boldsymbol{u} \\
& \dot{\alpha}_{P}=0 \\
& \dot{\boldsymbol{u}}_{P}=-\nabla\left(\alpha_{P} p_{P}\right) /\left(\rho Y_{P}\right) \\
& \dot{e}_{P}=\alpha_{P} p_{P} \nabla \cdot \boldsymbol{u}_{P} /\left(\rho Y_{P}\right) \\
& \dot{C}_{i}=0 \\
& \dot{Z}=0
\end{aligned}
$$

where subscript $i$ denotes each of the progress variables (HSR, M, QSB, GC). The gas velocity is found from the solid phase and total mixture velocity via. $\boldsymbol{u}_{g}=\left(\boldsymbol{u}-Y_{P} \boldsymbol{u}_{P}\right) / Y_{g}$. The species mass fractions are not transported because they are defined by the mixture fraction and reaction progress variables as described in Section 2.3.

During the second stage, the phase interactions source terms for the volume fraction and particle energy, and source terms for the reaction progress variables are integrated for the time increment while neglecting convective effects, and pressure work between the phases $\left(e . g .,-\left(p_{P}-\beta_{P}\right) V_{P}^{\ddagger}=0\right)$ resulting in the following ordinary differential equations (ODEs):

$$
\begin{aligned}
\frac{d \alpha_{P}}{d t} & =c_{P}^{\ddagger} / \gamma_{P} \\
\frac{d e_{P}}{d t} & =-\int \dot{q}_{P}^{\prime \prime} d A_{P} \\
\frac{d C_{i}}{d t} & =S_{i}
\end{aligned}
$$

A stiff ODE solver (Radhakrishnan and Hindmarsh, 1993) is used to integrate the resulting set of ODEs during this step. The momentum equation is not integrated during this stage because drag effects are incorporated using a semi-analytical solution. By neglecting bulk convective effects a semi-analytical solution of the form,

$\boldsymbol{u}_{s}^{t+\Delta t}=\boldsymbol{u}_{s}^{t} e^{-\frac{\Delta t}{\tau_{v e l}}}+\left(1-e^{-\frac{\Delta t}{\tau} v e l}\right) \boldsymbol{u}$

is used to relax the phase velocities. The quantity $\tau_{v e l}$ in Eq. (41) represents the characteristic time scale for velocity relaxation which is estimated as,

$$
\tau_{v e l}=\frac{8 Y_{s} \alpha_{s}}{\pi n_{d} \rho D_{P}^{2} C_{D}\left|\boldsymbol{u}_{s}-\boldsymbol{u}_{g}\right|}
$$

This semi-analytical solution eliminates the need to solve the momentum ODE in the stiff ODE solver.

The third stage is the pressure relaxation step, which accounts for the pressure work between the phases which is neglected during the phase interactions step. Assuming no convective effects, and mass transfer during this stage the following ODE is solved during this step:

$$
\begin{aligned}
& \frac{d \alpha_{P}}{d t}=\rho n_{P} V_{P}^{\ddagger} \\
& m_{P} \frac{d e_{P}}{d t}=-\left(p_{P}-\beta_{P}\right) V_{P}^{\ddagger}
\end{aligned}
$$


In Eq. (43b) the term $p_{P}-\beta_{P}$ represents the interface pressure between the phases. For all cases in this study the particle volume fraction is sufficiently low to neglect the configuration pressure (Carroll and Holt, 1973). Multiplying Eq. (43b) by $\rho n_{P}$ and replacing $p_{P}-\beta_{P}$ by the interface pressure $\left(p_{I}\right)$ results in,

$\rho_{P} \frac{d e_{P}}{d t}=-p_{I} \frac{d \alpha_{P}}{d t}$

which is the final form of the solid phase energy ODE solved during this step.

The pressure work terms were neglected during stages 1 and 2 due to the extremely small time scales associated with the pressure equilibrium compared to the other phase interaction processes. The characteristic time scale for a particle to equilibrate to the surrounding gas pressure can be estimated as $\tau_{\text {relax }, p}=N$ $D_{p} / c_{P}$, where $N \approx 3$ is a number of wave transversals for equilibration, and $c_{P}$ is the speed of sound in the solid. In general this time scale is of the order $10^{-8} \mathrm{~s}$ (Chinnayya et al., 2004). If these terms were included in the phase interactions step it would cause the system of ODEs to be excessively stiff.

During this step a pressure relaxation method is utilized in order to bring the phases into pressure equilibrium by adjusting the volume fractions and accounting for the resulting pressure work. A variety of different algorithms have been developed to accomplish this (Saurel and Abgrall, 1999; Chinnayya et al., 2004; Petitpas et al., 2009; Saurel et al., 2009; Benson, 1992; Lallemand et al., 2005), with varying efficiencies depending on the flow regimes and stiffness of the equation of states for each of the phases. The algorithm used in this study is given in Appendix A.

For the gas phase a Becker-Kistiakowsky-Wilson (BKW) equation of state (EOS), $p=\rho R T(1+X \exp (\beta X))$ is used where $X=$ $\left(\kappa \sum x_{i} k_{i}\right) /\left(V(T+\Theta)^{\zeta}\right)$, and $\beta, \kappa, \Theta$, and $\zeta$ are empirical parameters (Mader, 1998). The summation, $\sum x_{i} k_{i}$, is a mass weighted sum of the co-volumes over all the species in the gas phase. The values of the co-volumes for various species can be found in Hobbs and Baer (1992) and Mader (1998) and are, in general, a function of the initial condensed phase explosive. For the present study they are chosen to be $\beta=0.403, \kappa=10.86, \Theta=5442$, and $\zeta=0.499$ (Hobbs and Baer, 1992). The gas phase species considered are $\mathrm{O}_{2}$, $\mathrm{N}_{2}$, Al vapor, and $\mathrm{Al}_{2} \mathrm{O}_{3}$.

Considering separate EOSs for the aluminum and alumina is found to be important due to their different phase boundaries. A separate SESAME (Kerley, 1991) tabular equation of state is used for each of the solid constituents. In order to eliminate the need for additional transport equations, both solid constituents are considered to be in pressure, temperature, and velocity equilibrium. A Newton root finding method is used to find the equilibrium state given the solid phase mixture properties, as described in Appendix B.

\section{Results}

\subsection{Isolated particle}

Several isolated particle simulations are conducted to explore the validity of the current burn model and compare to the experimental data of Bazyn et al. $(2005,2007)$ and the detailed direct numerical simulations (DNS) of Washburn et al. (2010) that span a range of pressure and oxygen environments. Fig. 7 shows representative time series results of (a) particle temperature and normalized flame radius assuming an infinitely thin flame sheet that is computed as (DesJardin et al., 2005):

$r_{f} / r_{p}=\frac{1}{r_{p}}\left\{\frac{-4 \pi \mu_{g}}{S c_{g} \dot{m}_{P}} \ln \left[\frac{Y_{o x, \infty} / v_{o x}+f+1}{f+1}\right]\right\}^{-1}$

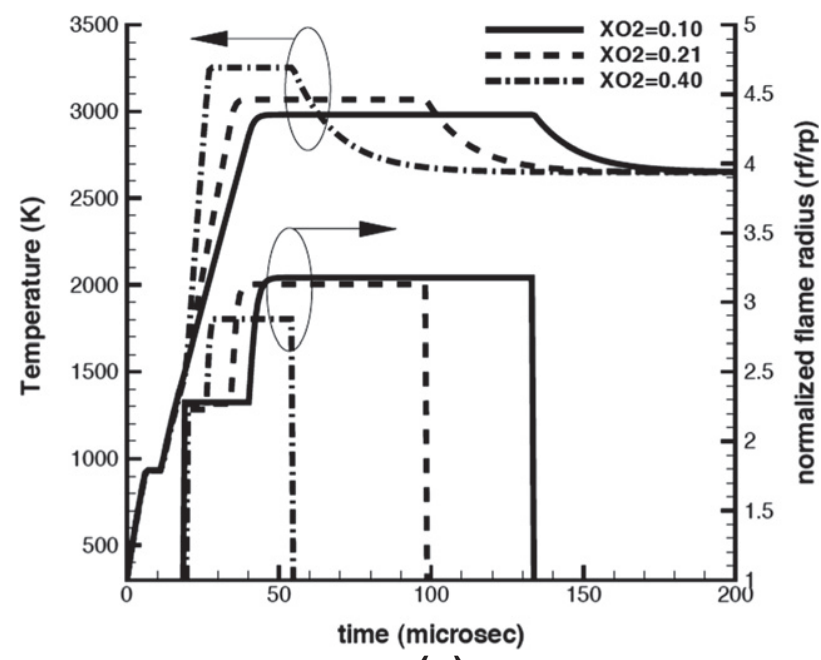

(a)

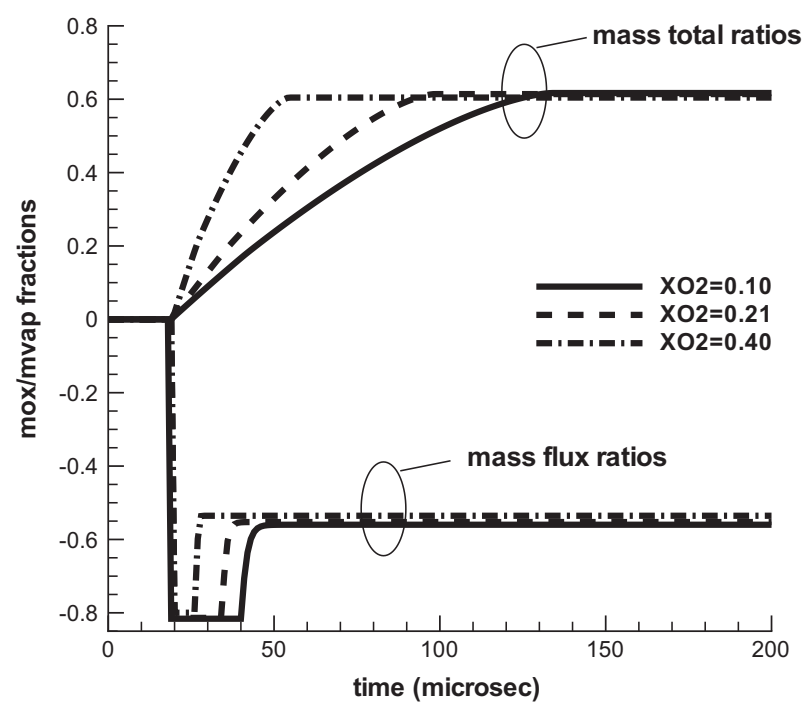

(b)

Fig. 7. Time series results of individual particle simulations showing (a) $T_{P}$ and $r_{f} / r_{p}$ and (b) $m_{m o x} / m_{P}^{0}$ and $\dot{S}_{\text {mox }}^{\prime \prime} / \dot{m}^{\prime \prime}$ for far-field oxygen mole fractions of $X_{O_{2}}=0.10$ 0.21 and 0.40 . For all cases $T_{\infty}=2650 \mathrm{~K}$ and $P_{\infty}=8.5 \mathrm{~atm}$.

and (b) ratio of metal oxide cap to initial mass $\left(m_{m o x} / m_{P}\right)$ and metal oxide deposition rate to the vapor mass flux $\left(\dot{S}_{\text {mox }}^{\prime \prime} / \dot{m}_{\text {vap }}^{\prime \prime}\right)$ for farfield oxygen mole fractions of $X_{\mathrm{O}_{2}}=0.10,0.21$ and 0.40 . For all cases, the initial temperature of the particle is set equal to $300 \mathrm{~K}$ and the far-field temperature and pressure are set equal to $2650 \mathrm{~K}$ and $8.5 \mathrm{~atm}$, respectively, to match the conditions used by Washburn et al. (2010). With increasing oxygen, the normalized flame radius decreases so as to match the required stoichiometric fuel loading into the flame, i.e., increasing far-field oxygen results in higher oxygen loading into the flame therefore the flame must decrease in order for a commensurate rise in the fuel loading. With the flame closer to the surface the particle temperature rises $200-300 \mathrm{~K}$ as the oxygen is reduced by a factor of two. Fig. $7 \mathrm{~b}$ shows the resulting changes in metal oxide cap formation from changes in the surrounding oxygen content. The lower set of curves shows $\dot{S}_{\text {mox }}^{\prime \prime} / \dot{m}_{\text {vap }}^{\prime \prime}$ is negative meaning the solid metal oxide is going to the particle surface and the vapor away. During the transition from stage I to II as the flame expands out, the metal oxide mass flux is nearly $80 \%$ that of the metal vapor. Once steady-state burning is achieved 


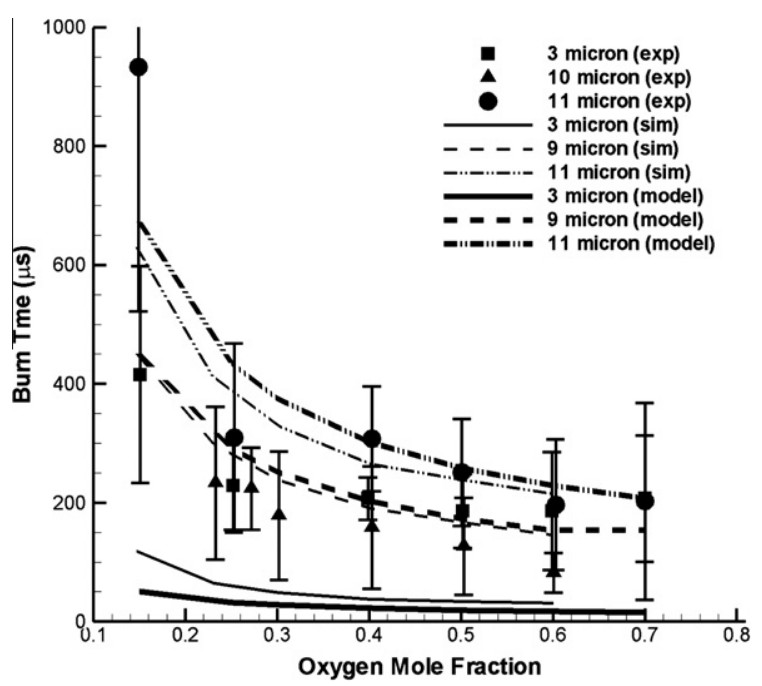

Fig. 8. Burn time vs. $X_{\mathrm{O}_{2}}$ for initial particle diameters of 3,10 (or 9) and $11 \mu \mathrm{m}$ particles. The symbols are data from Bazyn et al. $(2005,2007)$, the lines are the simulations of Washburn et al. (2010) and the bold lines are using the present model. For all cases $T_{\infty}=2650 \mathrm{~K}$ and $P_{\infty}=8.5 \mathrm{~atm}$.

and the flame reaches a position of $\sim 3$ particle radii then the metal oxide flux decreases to $58 \%$ of the metal vapor. Also shown in Fig. 7b is the overall metal oxide mass deposited on the particle as a fraction of the initial mass where $60 \%$ of the original mass diffuses back to the surface and forms a cap.

Fig. 8 shows comparisons of the burn times using the data from Bazyn et al. (2005, 2007) (symbols), the simulations of Washburn et al. (2010) (lines) and the present model (bold lines). The overall agreement of the model to the simulations is quite good with a maximum difference of $10 \%$ for the 9 and $11 \mu \mathrm{m}$ particle cases indicating that the model is capable of accounting for the leading order effects of far-field oxygen content on burn times. Slightly larger differences are observed for the $3 \mu \mathrm{m}$ case and is attributed to the simplified treatment of the oxide cap in the model. In the simulations of Washburn, an effective surface area for vaporization is computed to account for the presence of the oxide cap. The effect of the oxide cap on the evaporation rate is expected to be more pronounced with smaller particles where the oxide layer is a greater percentage of the overall volume of the particle. The complicated dynamics of the oxide cap on the particle are not accounted for in the present model and therefore explains the larger differences in burn times shown in Fig. 8.

Fig. 9 shows (a) surface temperature $\left(T_{s}\right)$ and $r_{f} / r_{p}$, and (b) $\dot{S}_{\text {mox }}^{\prime \prime} / \dot{m}_{\text {vap }}^{\prime \prime}$ and $m_{\text {mox }} / m_{P}$ for the same cases as Fig. 8. Consistent with the time series plots of Fig. 7, an increase in $\mathrm{O}_{2}$ results in a decrease in flame radius, increasing the surface temperature leading to the observed decrease in burn time. As shown in Fig. 9a values of $T_{s}$ for the high $\mathrm{O}_{2}$ cases are 200-300 K below the boiling temperature of $3364 \mathrm{~K}$ at these pressures - consistent with the findings of Washburn et al. (2010). Fig. 9b shows the sensitivity of the metal oxide cap to $\mathrm{O}_{2}$. An interesting local maximum is observed for $X_{\mathrm{O}_{2}}=0.06$ where nearly $58 \%$ of the original mass is converted to metal oxide (note, this value is somewhat lower than that in Fig. 7 of $60 \%$ since the time series plots include metal oxide formation from both HSR and quasi-steady burning whereas the results in Fig. 9 only include the contribution from quasi-steady burning).

The local maximum is from the competing effects of increased flame surface area and decreasing $T_{s}$ with increasing $\mathrm{O}_{2}$ levels. As $\mathrm{O}_{2}$ is decreased the flame surface area increases to balance the stoichiometric fuel loading into the flame thereby increasing the amount of metal oxide deposited back to the particle surface up until $X_{\mathrm{O}_{2}}=0.1$. Lower values of oxygen beyond this point result

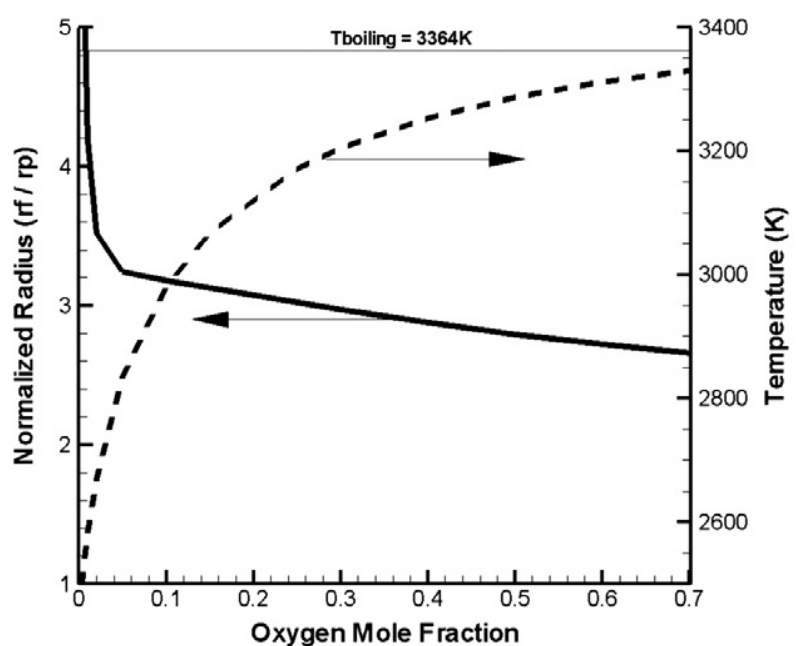

(a)

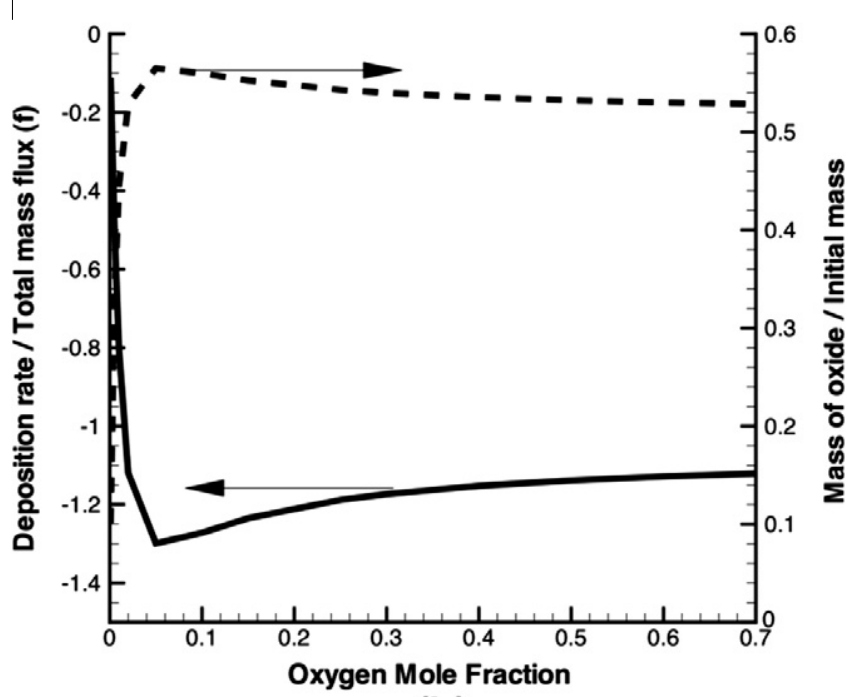

(b)

Fig. 9. Sensitivity of particle predictions to far-field $\mathrm{O}_{2}$ showing (a) $r_{f} / r_{p}$ and $T_{s}$ and (b) $\dot{S}_{\text {mox }}^{\prime \prime} / \dot{m}_{\text {vap }}^{\prime \prime}$ and $m_{\text {mox }} / m_{p}^{0}$. For all cases $T_{\infty}=2650 \mathrm{~K}$ and $P_{\infty}=8.5 \mathrm{~atm}$.

in a precipitous decrease in $T_{s}$ (Fig. 9a), increasing the flame radius which then serves to decrease the evaporation rate (via, the vapor pressure curve) and thereby decreasing the overall amount of metal oxide formed. For very low values of $\mathrm{O}_{2}$, the flame radius balloons out to very large non-physical values, indicating the start of flame extinguishment from $\mathrm{O}_{2}$ starvation.

Fig. 10 shows the sensitivity of the particle model burn times to pressure with the same comparisons as that of Fig. 8. The data and simulation show a transition from kinetics to diffusion controlled reactions at $P_{\infty} \simeq 10 \mathrm{~atm}$, while the model results do not. The reason for this is the current model does not contain finite rate chemistry and therefore is not able to account for the transition from kinetics to diffusion controlled reactions. However, the formulation does not preclude the incorporation of finite rate chemistry since it is based on a Shvab-Zel'dovich formulation - not a zonal description that assumes infinitely fast chemistry.

The thinness of the reaction zone is implicitly defined by the current assumption that the oxygen concentration at the particle surface is equal to zero which sufficiently constrains the resulting eigenvalue problem so a solution may be found (DesJardin et al., 2005). However, this constraint could be relaxed and the effects of finite-rate chemistry could be re-introduced using a finite rate 


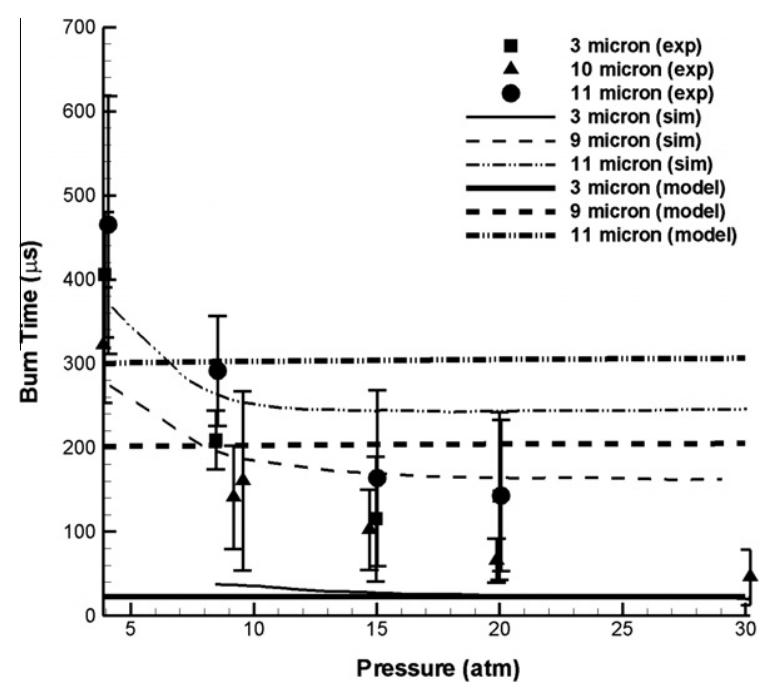

Fig. 10. Burn time vs. pressure for initial particle diameters of 3, 10 (or 9) and $11 \mu \mathrm{m}$ particles. The symbols are data from Bazyn et al. $(2005,2007)$, the lines are the simulations of Washburn et al. (2010) and the bold lines are using the present model. For all cases $T_{\infty}=2650 \mathrm{~K}$ and $X_{\mathrm{O}_{2}}=40 \%$.

chemistry model to account for surface reactions during the vapor stage of combustion.

Fig. 11 further illustrates the effect of pressure on the burning showing (a) $T_{s}$ and $r_{f} / r_{p}$, and (b) $\dot{S}_{\text {mox }}^{\prime \prime} / \dot{m}_{\text {vap }}^{\prime \prime}$ and $m_{\text {mox }} / m_{P}$ for pressures up to $10,000 \mathrm{~atm}$. Small particles can start to burn during the early stages of flow expansion where post-detonation products have pressures that range from 50,000 to 100,000 atm.

At these very high pressures, the flame radius is quite small since the vapor pressure is low on the surface relative to the surrounding pressure (i.e., low mass flux). Fig. 11 shows that the boiling temperatures at these high pressures can be well over $9000 \mathrm{~K}$ and the mass flux of metal oxide back to the surface is actually larger than the flux of metal vapor outward to the flame. According to the model, the resulting final metal oxide mass can exceed $80 \%$ of the original mass - indicating that the mox residue from burning may include some rather large caps compared to low pressure burning. Based on the assumed stoichiometry of the aluminum reaction, a maximum of $189 \%$ of mox can be formed in the cap if all of the mox formed in the flame diffuses back to the liquid surface. While these interesting result are consistent with the phenomenology of the model, it remains unproven that this type of burning can take place since, to the authors knowledge, there has not been detailed studies of burning aluminum particles at these very large pressures. Nonetheless, the model allows for these interesting limits to be explored.

\subsection{Explosives components facility tests}

\subsubsection{Experimental setup}

Several experiments were conducted at the Sandia National Laboratories Explosive Components Facility (ECF) to determine the behavior of the thermobaric explosive TBX, composed of isopropyl nitrate, RDX, aluminum flake, and Cab-o-sil $\left(\mathrm{SiO}_{2}\right)$. Cases were conducted for TBX charges located at the end of the ECF test chamber, and in the middle of the chamber. A cut-away illustration of the ECF test chamber configured for a charge located at the end is shown in Fig. 12.

The test charges are spherical and consist of nested hollow plastic shells with C-4 in the inner shell and TBX slurry in the outer shell. Each shell is split in half for filling and glued (inner) or taped (outer) back together. A RP-1 detonator is inserted into the C-4

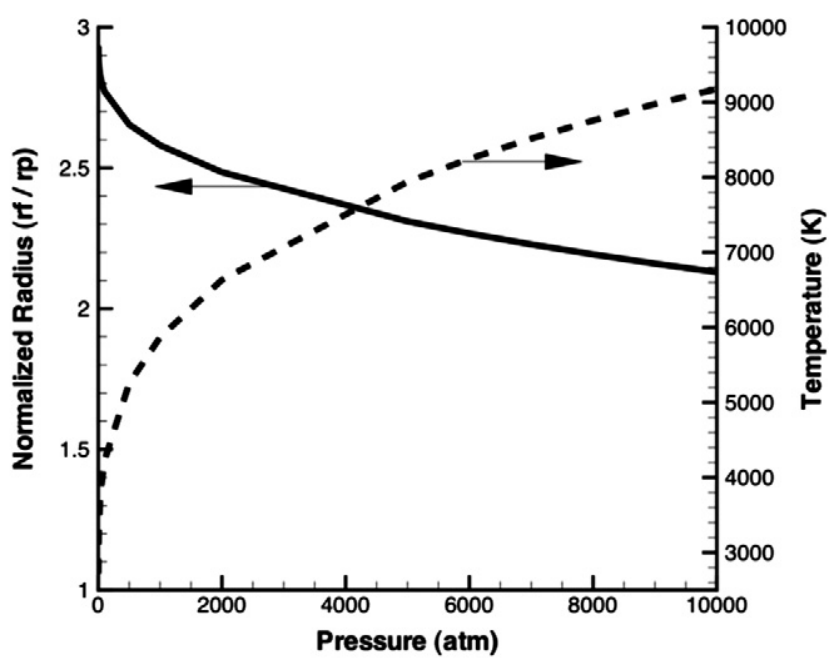

(a)

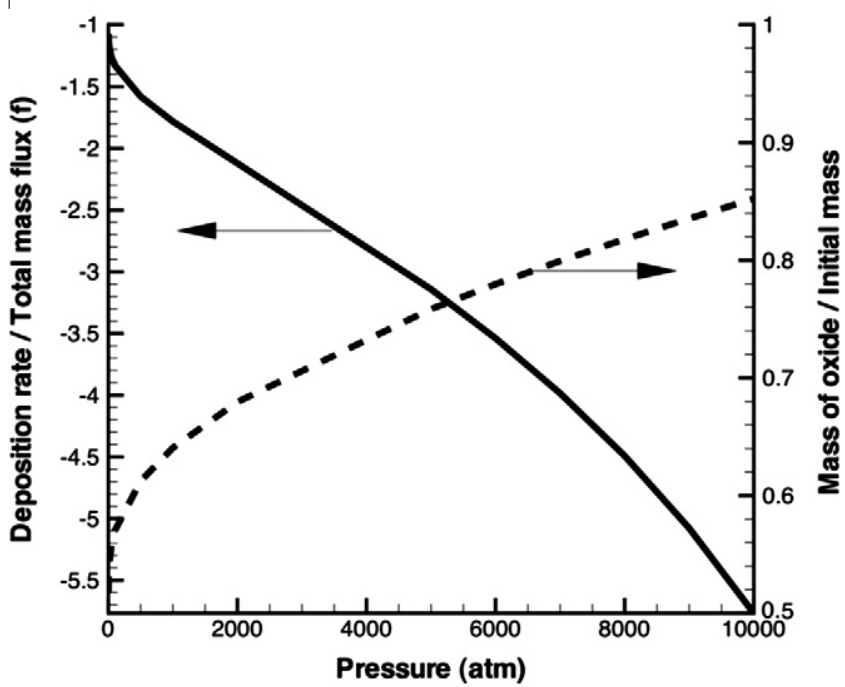

(b)

Fig. 11. Sensitivity of particle predictions to pressure showing (a) $T_{s}$ and $r_{f} / r_{p}$ and (b) $\dot{S}_{\text {mox }}^{\prime \prime} / \dot{m}_{\text {vap }}^{\prime \prime}$ and $m_{\text {mox }} / m_{p}^{0}$. For all cases $T_{\infty}=2650 \mathrm{~K}$ and $X_{\mathrm{O}_{2}}=40 \%$.

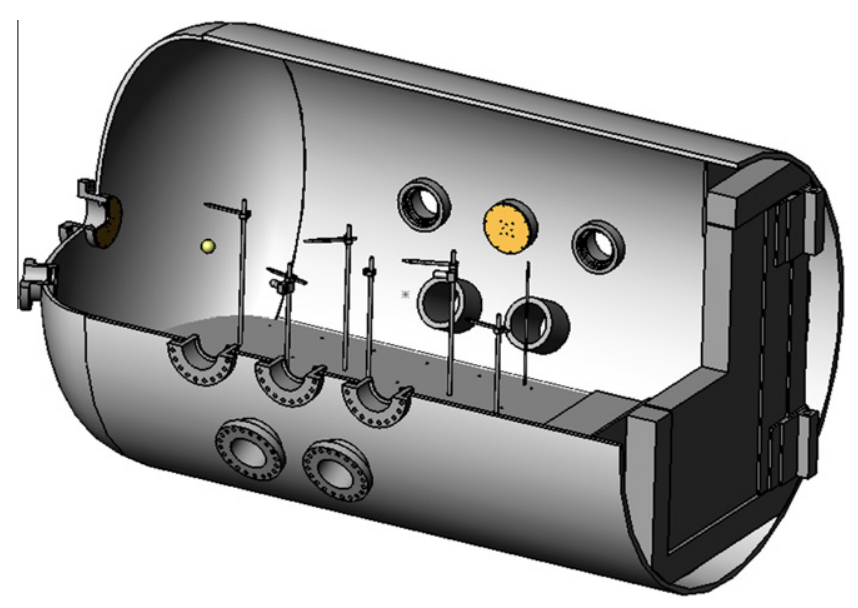

Fig. 12. Cutaway view of ECF test chamber showing the charge and diagnostic setup for a charge located at the end. 


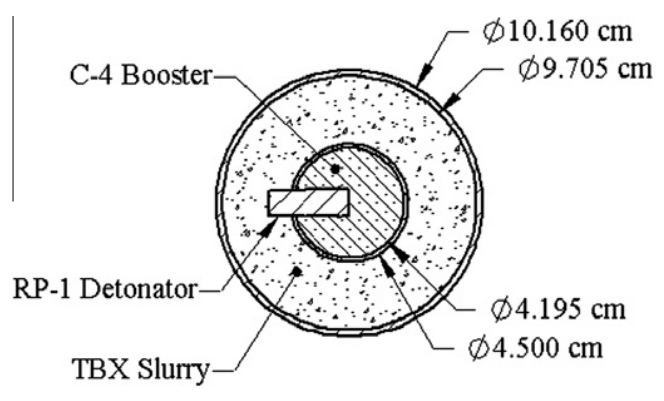

Fig. 13. Cross section of $650 \mathrm{~g}$ spherical TBX charge.

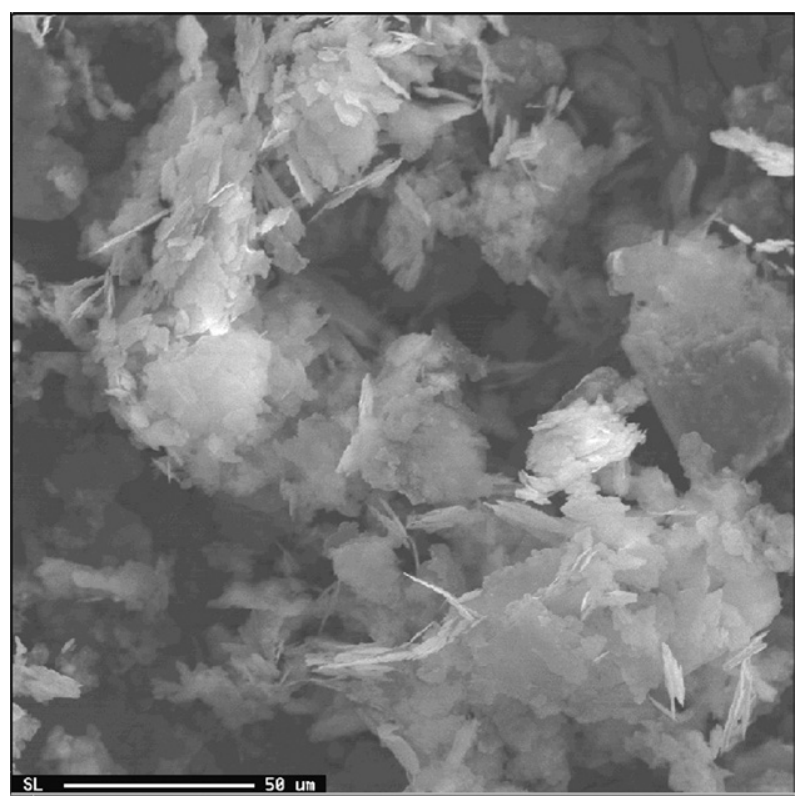

Fig. 14. Scanning electron microscope images of aluminum flakes used in thermobaric (TBX) mixture.

with its tip at the center. The charges have a $101.6 \mathrm{~mm}$ outside diameter with a $45 \mathrm{~mm}$ diameter booster. The booster nominally contains 61 grams of $\mathrm{C}-4$, and the main charge contains $589.7 \mathrm{~g}$ of TBX slurry. Fig. 13 shows a cross-sectional illustration of the spherical charge.

Pencil gauges were mounted inside the chamber usually pointing towards the charge to measure the pressure time history. The pencil gauges were not thermally protected. The pencil gauges used for all the cases compared to are manufactured by PCB (model $137 \mathrm{~A} 22$ ) with a 500 psi range, resonant frequency of greater than $500 \mathrm{kHz}$, a rise time of less than $4 \mu \mathrm{s}$, and a time constant of greater than $0.2 \mathrm{~s}$. The pencil gauges generally performed well as long as the cables were rugged and undamaged. The pencil gauge locations for the cases where the charge is located at the end and center are shown in Fig. 15.

For each charge location three experiments were conducted to ascertain the repeatability of the results. Additionally two experiments were conducted with the chamber flooded with $N_{2}$ in order to determine the degree of post-detonation product reactions with the surrounding excess oxygen. Overall good repeatability is seen in the pencil gauge histories.

\subsubsection{Problem setup}

In CTH the TBX in the outer shell is simulated by using the multiphase aluminum combustion model. The initial condition for the mixture is determined by specifying the initial density to be that of the TBX slurry $\left(1.37 \mathrm{~g} / \mathrm{cm}^{3}\right)$, and a gas temperature of $2224 \mathrm{~K} \mathrm{calcu-}$ lated by the CHEETAH (Fried and Souers, 1994) equilibrium thermodynamics code. This specifies the initial thermodynamic state for the gas. Assuming mechanical equilibrium between the solid and gas phases allows for the determination of the initial density of the aluminum particles from the Hugoniot relation,

$p_{H}\left(\rho_{s}\right)=p_{0}+\frac{\mu}{(1-S \mu)^{2}} \gamma_{0} c_{0}^{2}$

where $p_{0}, \gamma_{0}$, and $c_{0}$ are the reference pressure ( $\left.1 \mathrm{~atm}\right)$, density $\left(2.70 \mathrm{~g} / \mathrm{cm}^{3}\right)$, and speed of sound $(6420 \mathrm{~m} / \mathrm{s})$ for the metal where an assumed linear Us-Up curve is assumed with slope $S$. The quantity $\mu=1-\rho_{0} / \rho$ defines the extent of compression of the post-detonated metal. Setting $p_{H}$ equal to the post-detonated pressure from the explosive results in a quadratic equation for $\mu$ that is solved and used to initialize the temperature and energy of the particles.

The post detonation composition of the mixture is estimated using CHEETAH (Fried and Souers, 1994) using $\Phi=0.44$ and $F=1.0$ to best match the mass fraction of aluminum in the TBX and assuming that all the oxygen in the post detonation product species is free to react with the aluminum. A sensitivity study is conducted in Section 4.3 to determine the equivalence ratios effect on the model. A particle diameter of $30 \mu \mathrm{m}$ is chosen to match the flake size used in the TBX. The spherical particles are an approximation to the actual aluminum flake used in the experiments, shown in Fig. 14, where a distribution of flake sizes are present which is not considered in this study. Once the flakes melt surface tension should force the aluminum into an approximately spherical shape consistent with the spherical particles assumed in the model. The sensitivity of the model to initial particle diameter is explored in Section 4.3. From the initial particle size and equivalence ratio the initial solid volume fraction is determined. This completely specifies the initial state of the mixture. The C- 4 booster and detonator were not modeled and the outer and inner shells were neglected because they are assumed to have no significant effect on the results.

The chamber, including the floor, is simulated using a $300 \times$ $200 \times 2003 \mathrm{D}$ mesh, giving a mesh resolution of $1.6 \mathrm{~cm} \times 1.44 \mathrm{~cm}$ $\times 1.44 \mathrm{~cm}$. Fig. 16 shows pressure response near the explosive with increasing mesh refinement showing the results are mesh independent for grids greater than $300 \times 200 \times 200$, therefore this grid is used in all cases. A rigid material boundary is used for the chamber walls. Fixed tracers at the same locations as the pencil gauges were used in the simulations to compare the pressure histories to the experimental data. The simulations were conducted using 512 processors for nominally 3 days of computational time.

\subsubsection{Experimental comparisons}

Fig. 17 shows contour plots of the pressure field at the midplane of the chamber for $\Phi=0.44$ and $D_{P, o}=30 \mu \mathrm{m}$ at times of 3 , 6 , and $10 \mathrm{~ms}$. At $3 \mathrm{~ms}$ the initial blast wave from the charge has reflected off the elliptical end of the chamber, and by $6 \mathrm{~ms}$ the blast wave has reflected off the side walls of the chamber. By $10 \mathrm{~ms}$ the blast wave has reflected off the flat end of the chamber and several shock interactions and reflections are seen in the chamber.

Figs. 18 and 19 show the simulated pressure histories compared to the experimental data for pencil gauges 1-4 up to $10 \mathrm{~ms}$ for the charge located near the elliptical end and center of the chamber, respectively. Pencil gauge 3 history is not shown for experiment 2 in Fig. 19 due to a malfunction which resulted in no data being recorded. Also shown in Fig. 18 are pressure histories for simulations where the particles are allowed to melt and solidify but do not undergo HSR, QSB, or GC to show the effect of chemical reactions on the solution. The agreement of the data and simulation 


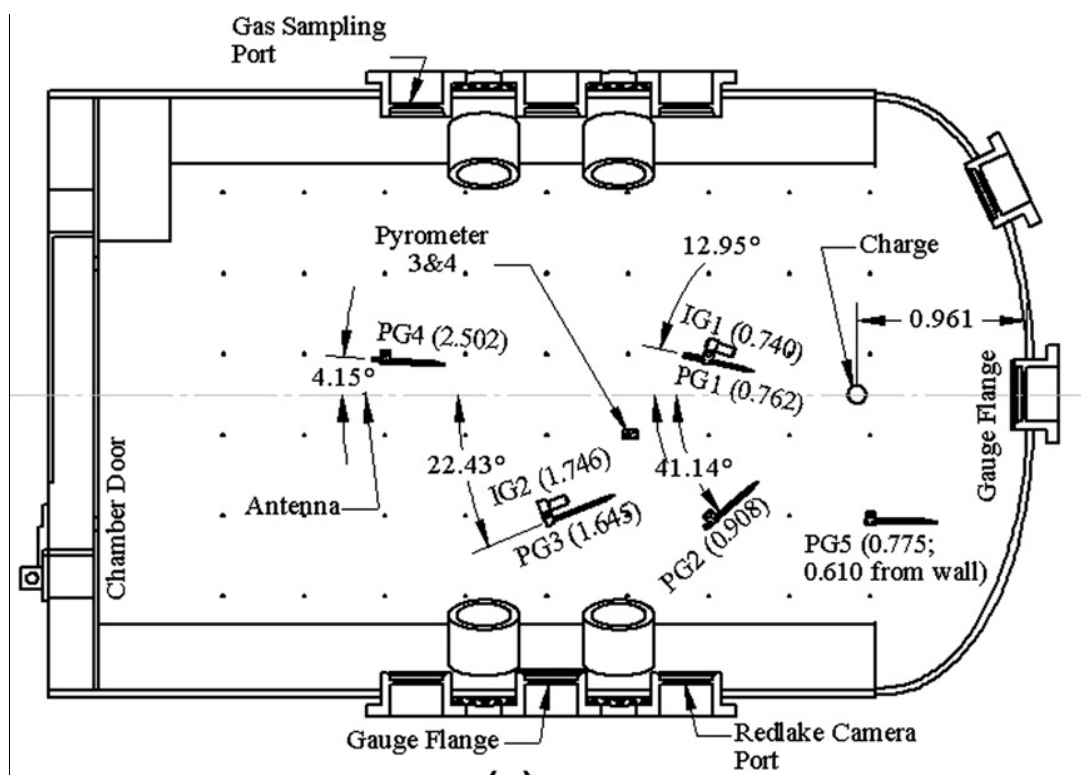

(a)

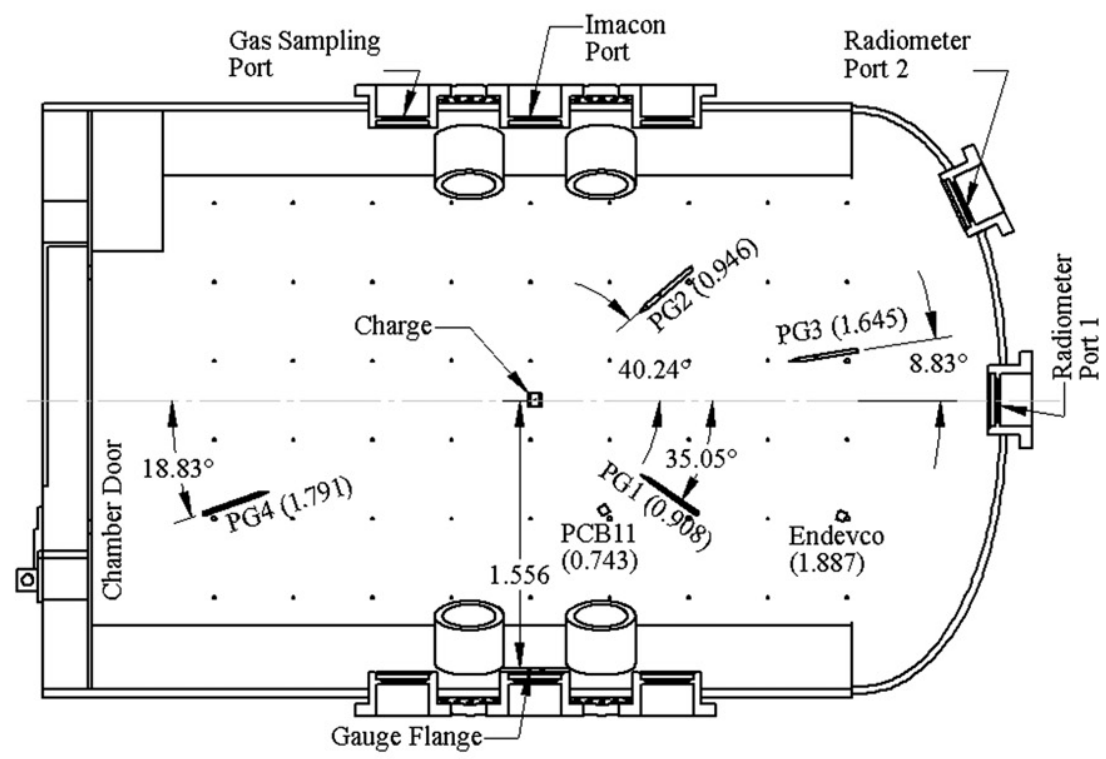

(b)

Fig. 15. Pencil gauge layout used for charges located at the (a) end, and (b) center of the chamber.

are excellent for most data points, and the timing of the pressure waves are accurately predicted. For some of the tracers the simulated initial blast wave is under predicted (by a maximum of $41 \%$ for pencil gauge 3 with the charge located at the center). This is attributed to uncertainties in setting the initial post-detonation mixture properties. Reactive mesoscale level multi-material simulations are needed in order to more accurately define the average post detonation properties of the charge, but this was not explored in the present study. The non-reactive simulation pressure histories in Fig. 18 show the importance of accounting for the aluminum reactions, without them the pressure magnitudes are under predicted by approximately $50-80 \%$.

Tables 1 and 2 compare the average experimental and simulated pressure impulses at the pencil gauges for charges located at the end and center of the chamber, respectively. The pressure impulse is under predicted for by $3.8-8.5 \%$ and is attributed to uncertainties in the initial post detonation mixture state predicted by CHEETAH, and uncertainty in the initial particle size.

For thermobaric high explosives such as TBX the reactions can exhaust the remaining oxygen from the charge after detonation, and if enough particles are present they can begin to use the oxygen in the surrounding atmosphere for combustion. In order to assess the effects of this on the behavior of the TBX, experiments were conducted with the charge located at the end of the chamber and the chamber flooded with $\mathrm{N}_{2}$, to prevent any reactions with the surrounding atmosphere. Oxygen is still present in the post detonation products of the TBX. Simulations are conducted for these cases and the pressure histories are compared in Fig. 20 along with the simulation results for the charge in air. Excellent agreement to the experimental data is shown and the pressure wave timings are accurately predicted. Minimal differences are seen between the air and $\mathrm{N}_{2}$ cases because the initial charge is 


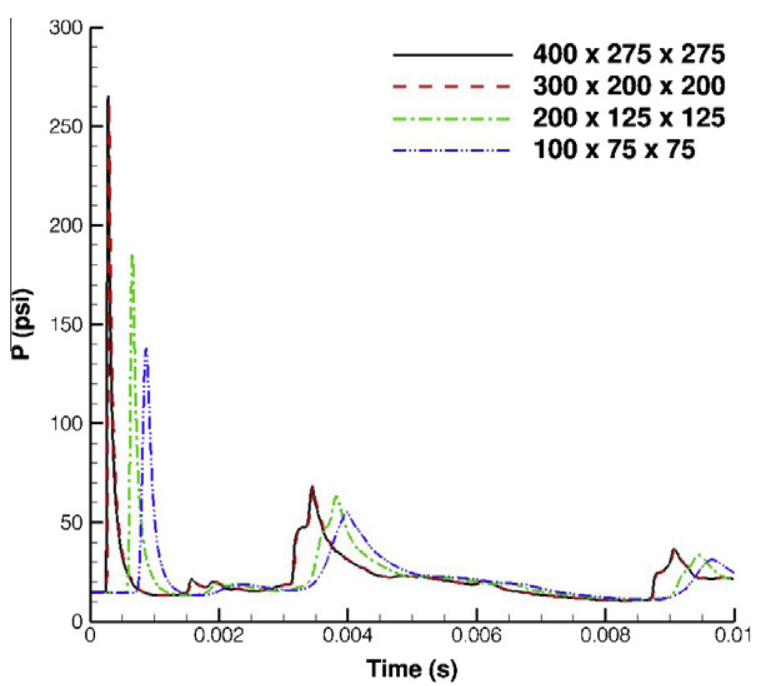

Fig. 16. Early time pressure response with increasing mesh refinement.

assumed to be fuel lean therefore all the aluminum in the initial charge is consumed and the excess oxygen surrounding the charge for the air cases is not needed. The magnitude of the pressure waves for the simulations with air are approximately $1-3 \%$ higher compared to the $\mathrm{N}_{2}$ simulations.

\subsection{Sensitivity studies}

Sensitivity studies are conducted in order to determine the influence of the initial particle diameter on the model. Lagrangian tracers are used to examine the pressure, temperature, and progress variable histories for a particle. Particle diameters of 3,30 , and $300 \mu \mathrm{m}$ are chosen with a constant equivalence ration of $\Phi=0.44$. Lagrangian tracer particles positioned on the outside surface of the charge facing directly towards the elliptical end of the chamber and door are examined.

Fig. 21 shows the pressure and melting progress variable histories, and temperature and quasi-steady burning progress variable for a tracer initialized on the outside of the charge towards the elliptical end for varying initial particle diameters. Fig. 22 shows the particle temperature and quasi-steady burning progress variable for the same tracer. As the particle size decreases the pressures increases due to the larger particle surface area resulting in more burning. The 3 and $30 \mu \mathrm{m}$ particles rapidly melt and combust whereas the $300 \mu \mathrm{m}$ particles only reach a maximum of $88 \%$ melted and never transition to quasi-steady burning due to their larger thermal inertia. The jump in pressure and temperature at approximately $4 \mathrm{~ms}$ is associated with the reflection from the elliptical end which serves to further heat and melt the $300 \mu \mathrm{m}$ particles. For the $300 \mu \mathrm{m}$ particles the initial expansion of the charge is delayed by $0.75 \mathrm{~ms}$ compared to the smaller particle cases because the initial post detonation pressure is $61 \%$ lower when compared to the $3 \mu \mathrm{m}$ particles because the $300 \mu \mathrm{m}$ particles do not ignite. For the particles that do ignite, a maximum value of $C_{O S B}=\Phi=0.44$ is achieved and is consistent with the fuel lean limit discussed in Section 2.3.4.

Fig. 23 shows the short time histories up to $0.2 \mathrm{~ms}$ of the pressure and melting progress variable for a tracer facing towards the elliptical end. Fig. 24 shows the particle temperature and quasisteady burning progress variable for the same tracer. For the $300 \mu \mathrm{m}$ particles the lower pressure and delayed expansion are clearly shown. The smaller particles undergo melting and quasi-

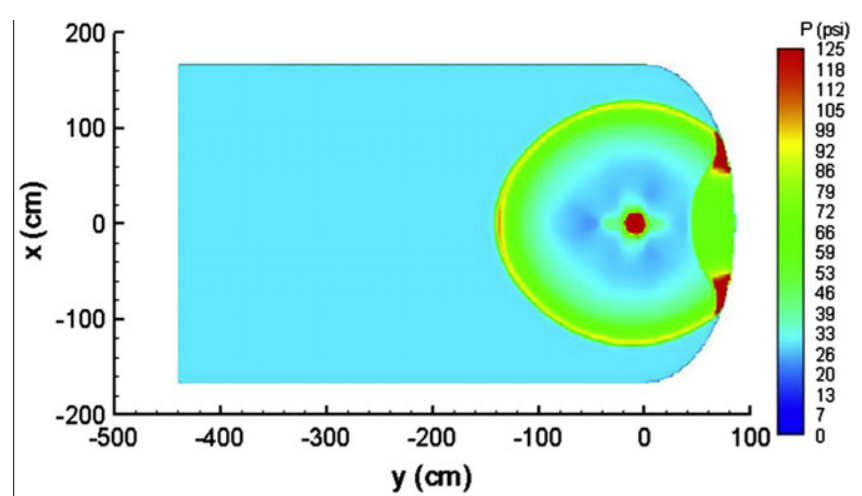

(a)

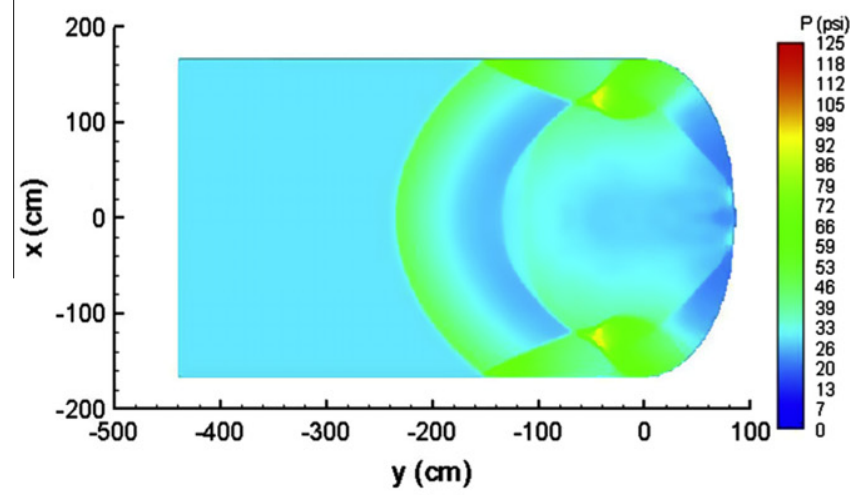

(b)

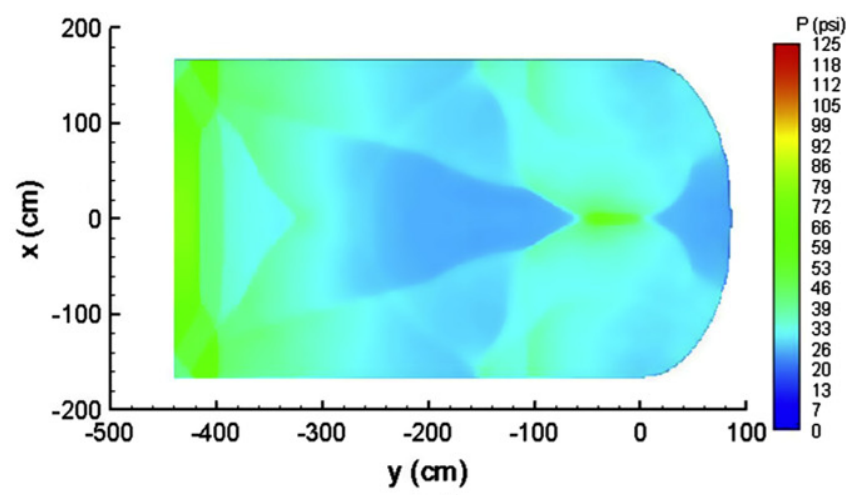

(c)

Fig. 17. Simulated pressure contours through midplane of chamber for $\Phi=0.44$, and $D_{P}=30 \mu \mathrm{m}$ at (a) $3 \mathrm{~ms}$, (b) $6 \mathrm{~ms}$, and (c) $10 \mathrm{~ms}$.

steady burning during the first $6 \mu$ s of the simulation while the $300 \mu \mathrm{m}$ particles are only $1 \%$ melted by the same time. The particle temperature during the initial charge expansion quickly reaches $5453 \mathrm{~K}$ for the $3 \mu \mathrm{m}$ diameter particles then decreases due to expansion. The very high simulated particle temperatures are due to the vapor pressure curve used in the aluminum particle model (Eq. (24)) which predicts a boiling temperature of over $9000 \mathrm{~K}$ for 10,000 atms for aluminum. This very high vaporization temperature at extreme pressures has also been predicted in previous research (Krieger, 1970).

Fig. 25 shows the pressure and melting progress variable for the same range of initial particle diameters for a tracer initialized on the outside of the charge facing the door of the chamber, opposite the previously examined tracer. Fig. 26 shows the temperature and 


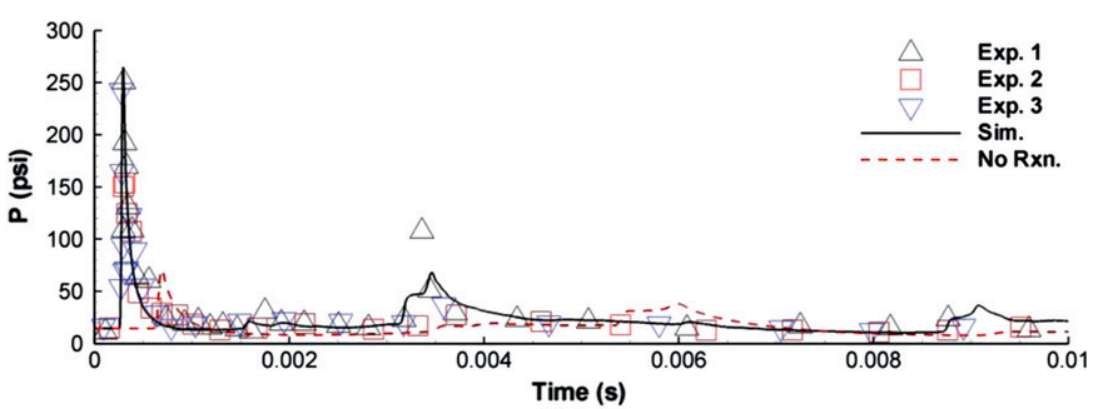

(a) Pressure history at pencil gauge 1

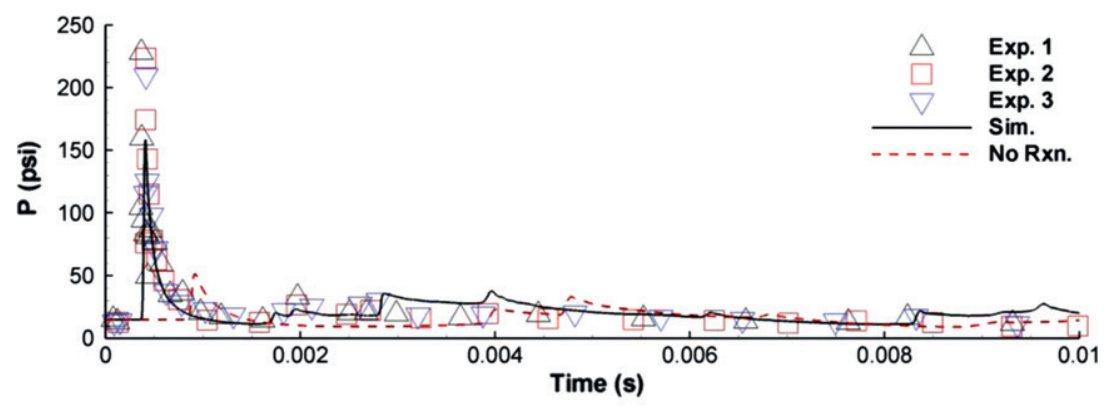

(b) Pressure history at pencil gauge 2

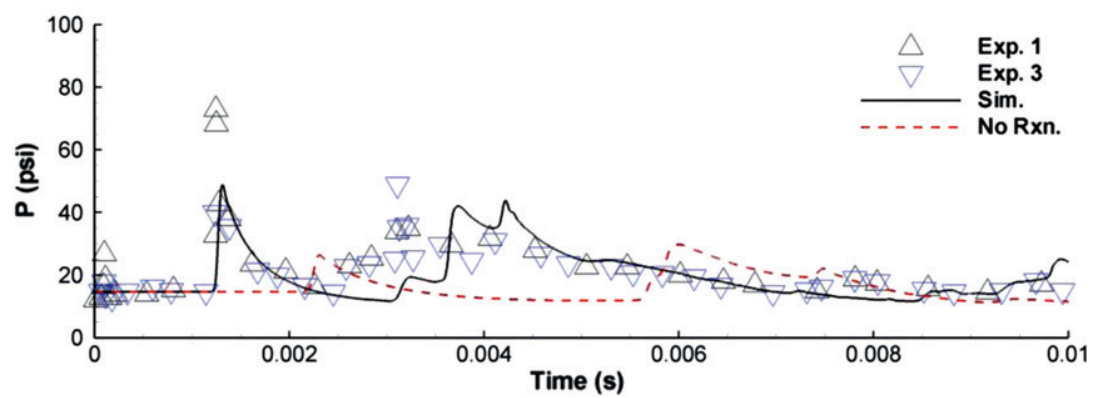

(c) Pressure history at pencil gauge 3

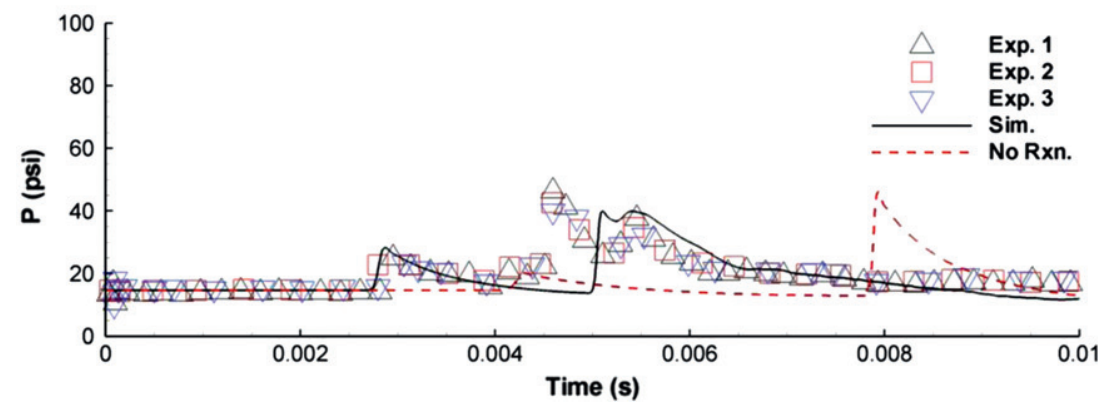

(d) Pressure history at pencil gauge 4

Fig. 18. Comparison of $\mathrm{CTH}$ results and ECF chamber test with charge located at the center for pencil gauges 1-4.

quasi-steady burning progress variable for the same conditions. The smaller 3 , and $30 \mu \mathrm{m}$ particles again burn during the initial expansion while the $300 \mu \mathrm{m}$ particles continue to solidify because of the lack of the strong reflection from the elliptical end, in contrast to the previous tracer behavior where they melted further after the reflection. The initial expansion for the $300 \mu \mathrm{m}$ particles is again significantly delayed compared to the smaller particles.

Sensitivity studies are also conducted to determine the effect of the initial charge equivalence ratio on the model. Fig. 27 shows the pressure and group combustion progress variable for initial equiv- alence ratios of $0.5,1,1.5$, and 15 . The group combustion progress variable rapidly increases to 1 for the fuel rich cases as the local oxygen around the particle is exhausted and the particles react with the excess oxygen surrounding the charge. The pressure impulse, $I=\int p d t$, as a function of initial equivalence ratio is shown in Fig. 28 for a tracer facing the elliptical end of the chamber. As the equivalence ratio increases, the impulse increases from the increase in overall heat release during combustion, until a maximum is reached for which an optimum amount of aluminum is present. This maximum is found to be between $\Phi=3$ and $\Phi=5$, which 


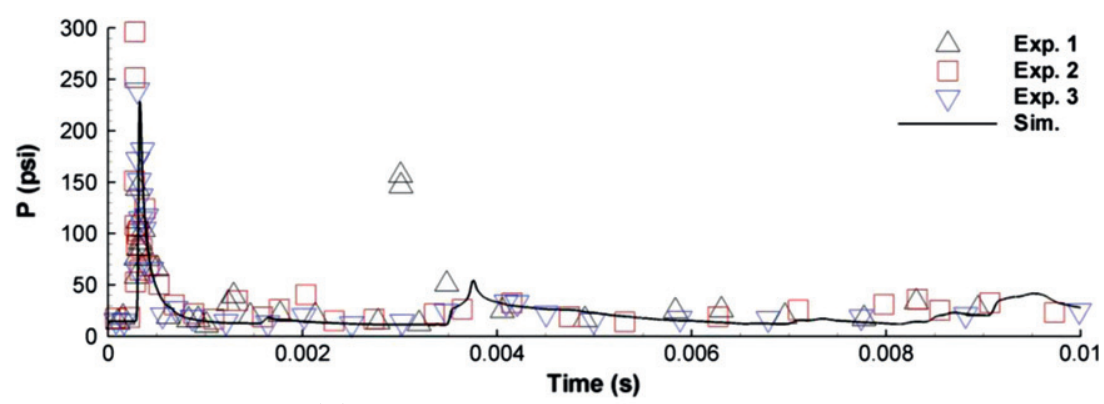

(a) Pressure history at pencil gauge 1

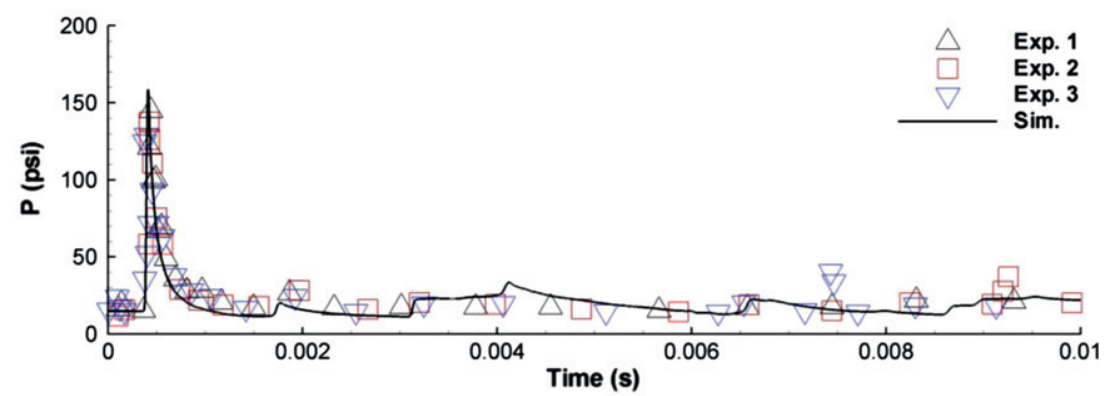

(b) Pressure history at pencil gauge 2

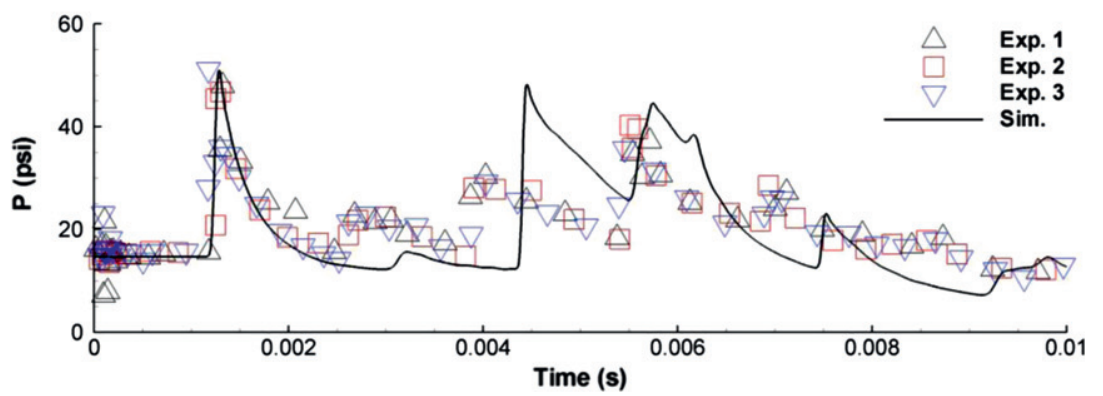

(c) Pressure history at pencil gauge 3

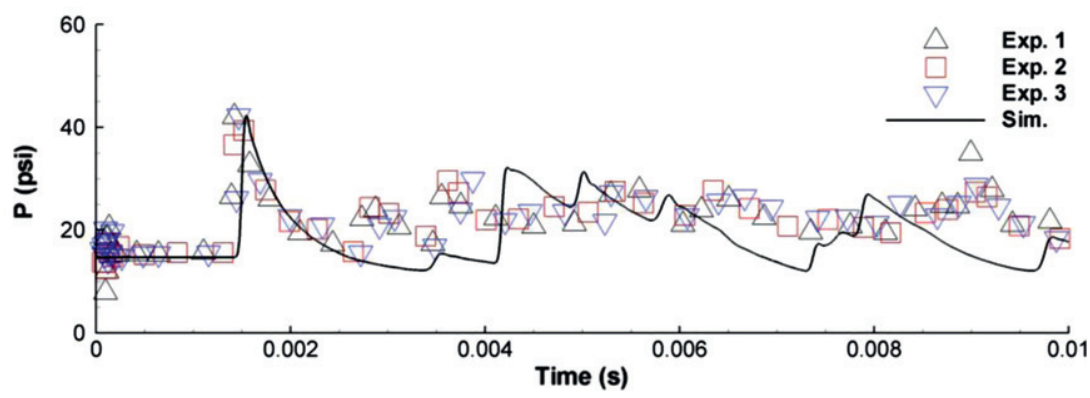

(d) Pressure history at pencil gauge 4

Fig. 19. Comparison of $\mathrm{CTH}$ results and ECF chamber test with charge located at the center for pencil gauges 1-4.

Table 1

Comparison of experimental and simulated impulses at pencil gauges 1-4 for charge located near the elliptical end of chamber.

\begin{tabular}{llll}
\hline Pencil gauge & Exp. avg. impulse $(p s i s)$ & Sim. impulse $(p s i s)$ & Error $(\%)$ \\
\hline 1 & 0.2346 & 0.2238 & -4.6 \\
2 & 0.2179 & 0.2097 & -3.8 \\
3 & 0.2054 & 0.1972 & -4.0 \\
4 & 0.2332 & 0.2134 & -8.5 \\
\hline
\end{tabular}

Table 2

Comparison of experimental and simulated impulses at pencil gauges 1-4 for charge located at the center of the chamber.

\begin{tabular}{llll}
\hline Pencil gauge & Exp. avg. impulse (psis) & Sim. impulse (psis) & Error $(\%)$ \\
\hline 1 & 0.4705 & 0.4381 & -6.89 \\
2 & 0.4728 & 0.4591 & -2.90 \\
3 & 0.4601 & 0.4402 & -4.33 \\
4 & 0.4593 & 0.4358 & -5.14
\end{tabular}




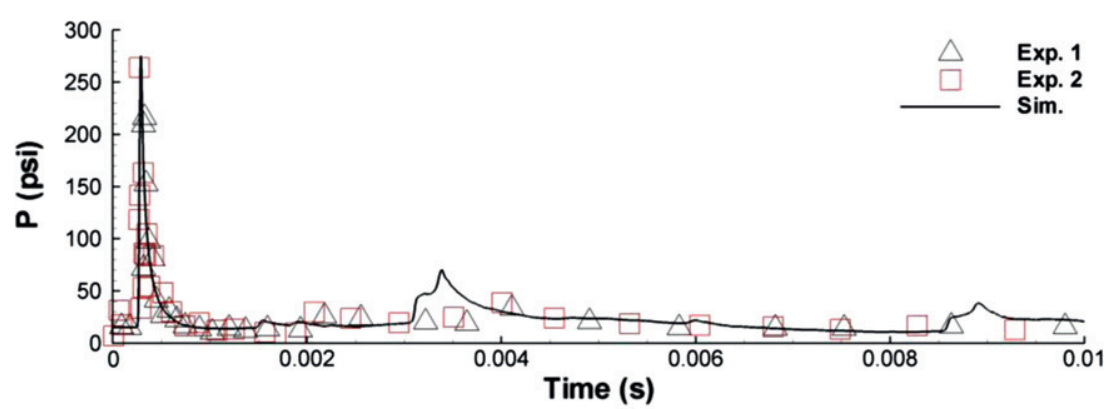

(a) Pressure history at pencil gauge 1

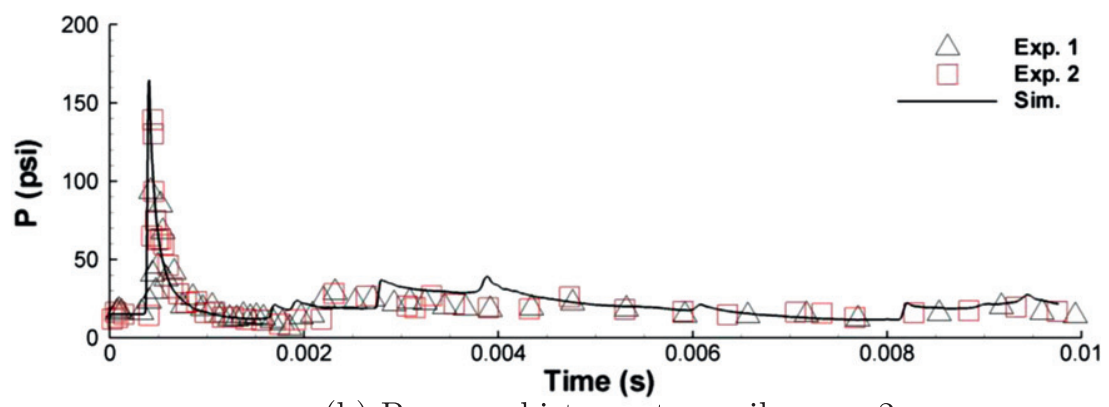

(b) Pressure history at pencil gauge 2

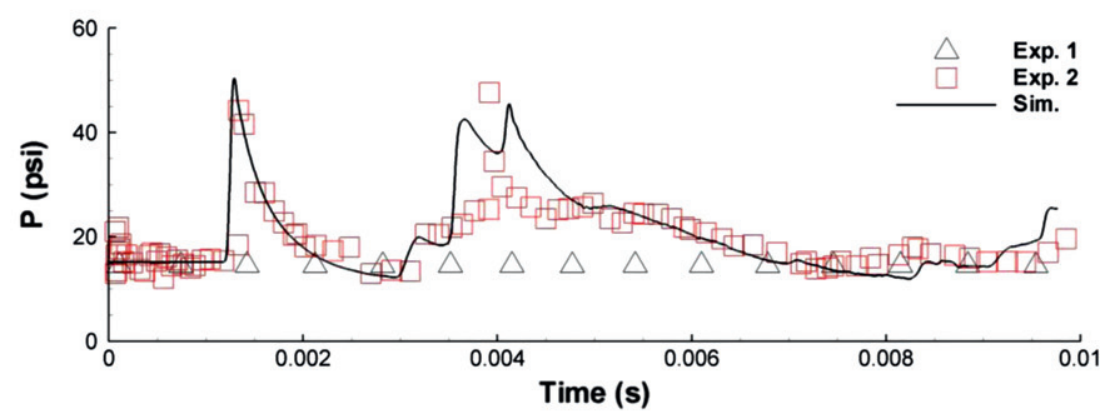

(c) Pressure history at pencil gauge 3

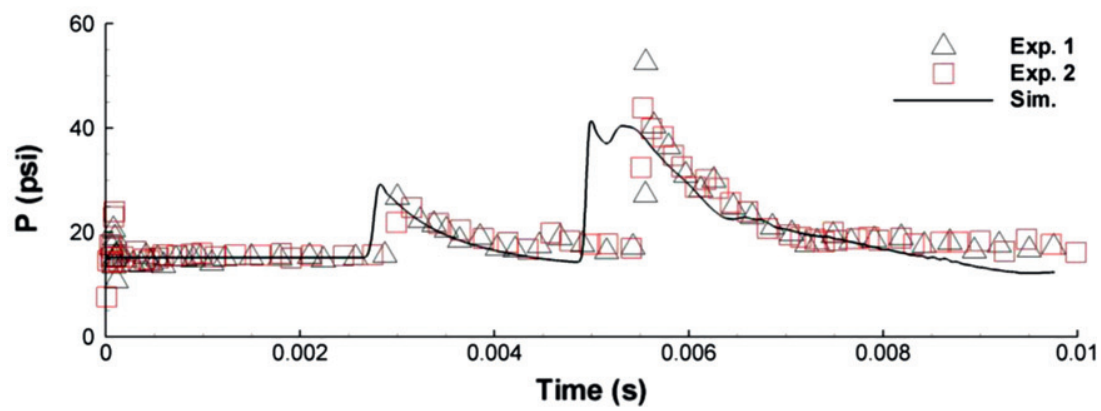

(d) Pressure history at pencil gauge 4

Fig. 20. Comparison of $\mathrm{CTH}$ results and ECF chamber test with charge located at the end and chamber filled with $\mathrm{N}_{2}$ for pencil gauges $1-4$

shows that mixing and reactions with the excess oxygen in the chamber are important to maximize the impulse. As the equivalence ratio is further increased to a value of 15 , the impulse decreases due to the inability of the oxygen from the air to mix with the fuel rich charge.

\section{Conclusions}

A reactive two-phase flow model and reaction progress variable formulation for aluminum particle combustion is developed and implemented into the CTH hydrodynamics code to simulate nonideal thermobaric explosives. A pressure relaxation approach is used to overcome the stiffness of the governing PDEs due to solid phase compressibility. The reaction progress variable approach allows the Lagrangian single particle model to be implemented in the Eulerian CTH code, and captures the effects of group combustion for aluminum rich explosives. In the future the formulation could be extended to account for the effects of unresolved SGS turbulence via single point joint PDF descriptions and effects of resolved viscous processes included. The modeling methodologies presented in this study could be applied to other shock driven high 


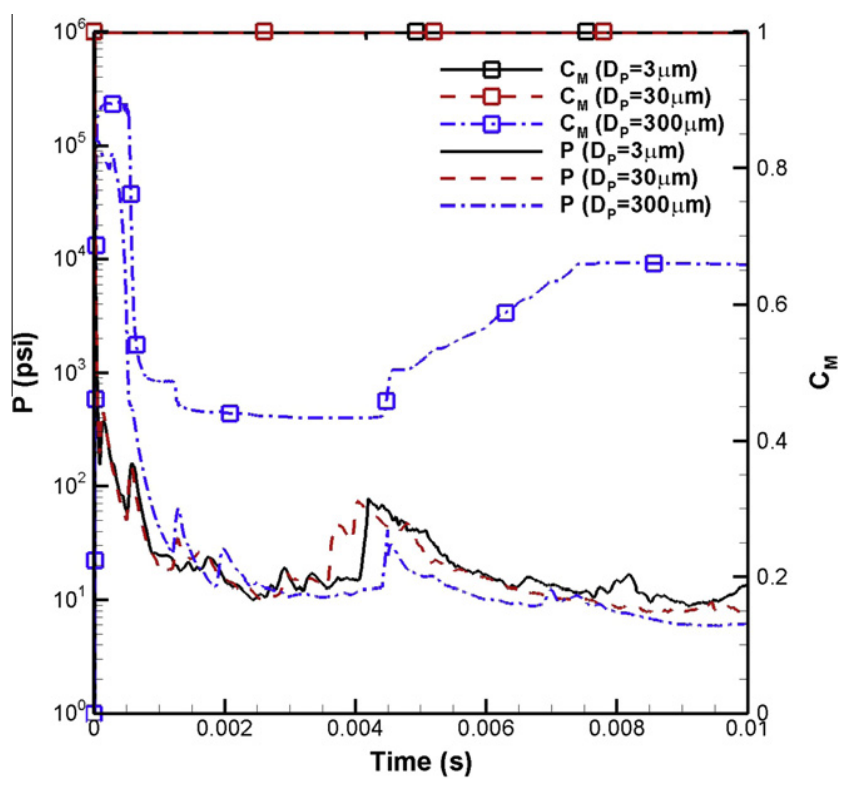

Fig. 21. Pressure and melting progress variable histories for Lagrangian tracer facing the elliptical end for initial particle diameters of $3 \mu \mathrm{m}, 30 \mu \mathrm{m}$, and $300 \mu \mathrm{m}$.

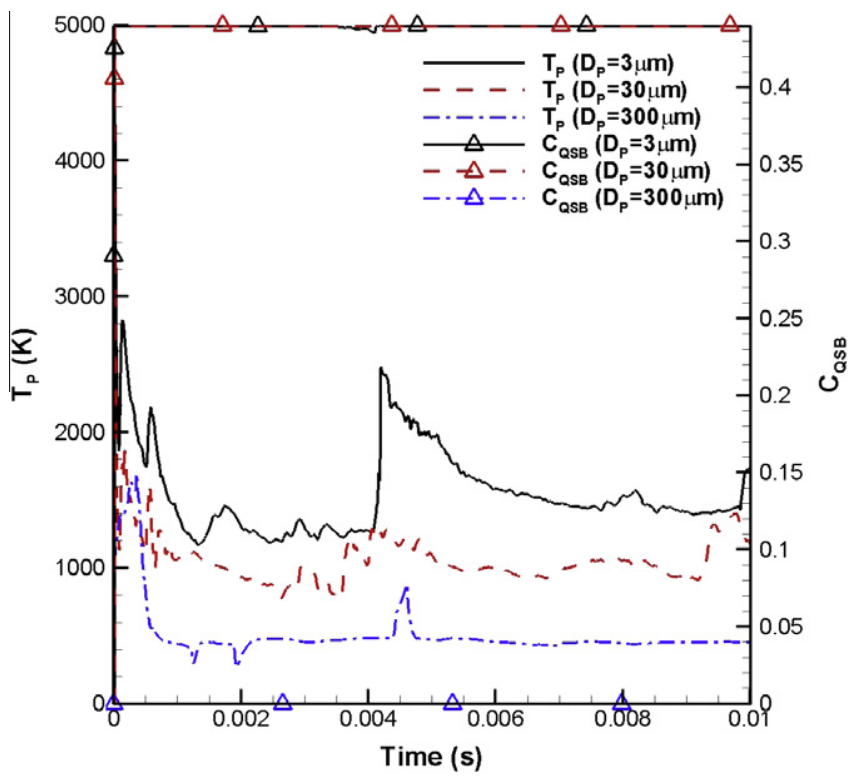

Fig. 22. Temperature and quasi-steady burning progress variable histories for Lagrangian tracer facing the elliptical end for initial particle diameters of $3 \mu \mathrm{m}$, $30 \mu \mathrm{m}$, and $300 \mu \mathrm{m}$.

pressure environments, such as hydrocarbon or coal combustion, and other dispersed particulates.

Isolated single aluminum particle results are compared to the experimental data of Bazyn et al. $(2005,2007)$ and the DNS of Washburn et al. (2010), and show reasonably good agreement for predictions of burn times as a function of oxygen mole fraction and pressure with a residue cap accounting for upwards of $60 \%$ of the original particle mass. The diffusion limited combustion regime is captured for single particle burn times as a function of pressure, but the kinetically limited regime is not observed by the current formulation due to the lack of finite rate chemistry in the model - a topic for future extensions.

Reactive multiphase simulations are conducted and compared to experimental results from the ECF blast chamber for TBX, show-

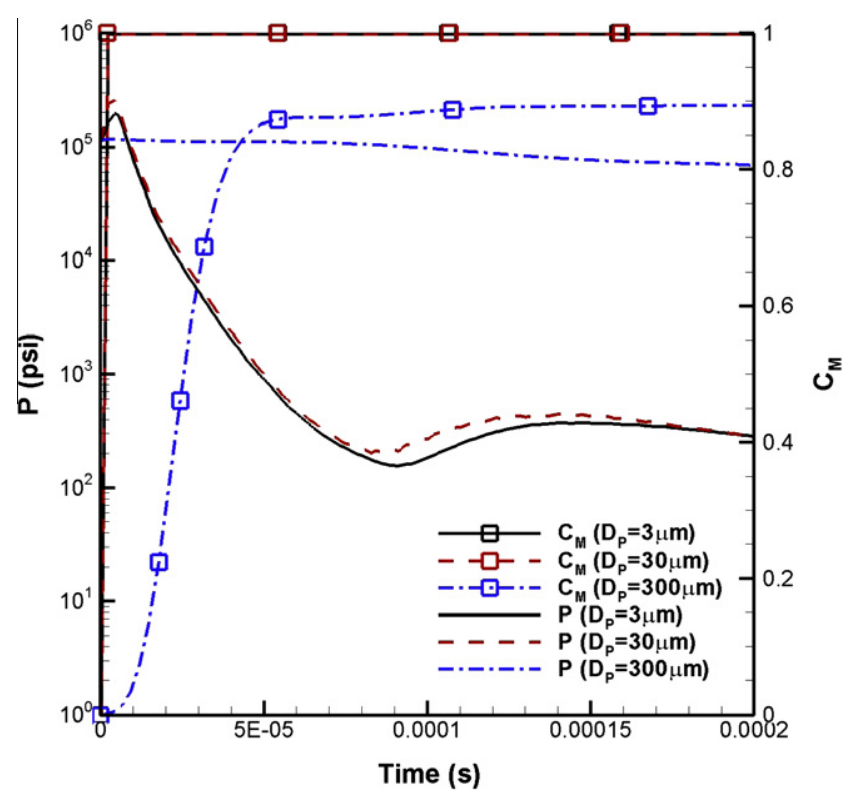

Fig. 23. Pressure and melting progress variable short time histories for Lagrangian tracer facing the elliptical end for initial particle diameters of $3 \mu \mathrm{m}, 30 \mu \mathrm{m}$, and $300 \mu \mathrm{m}$.

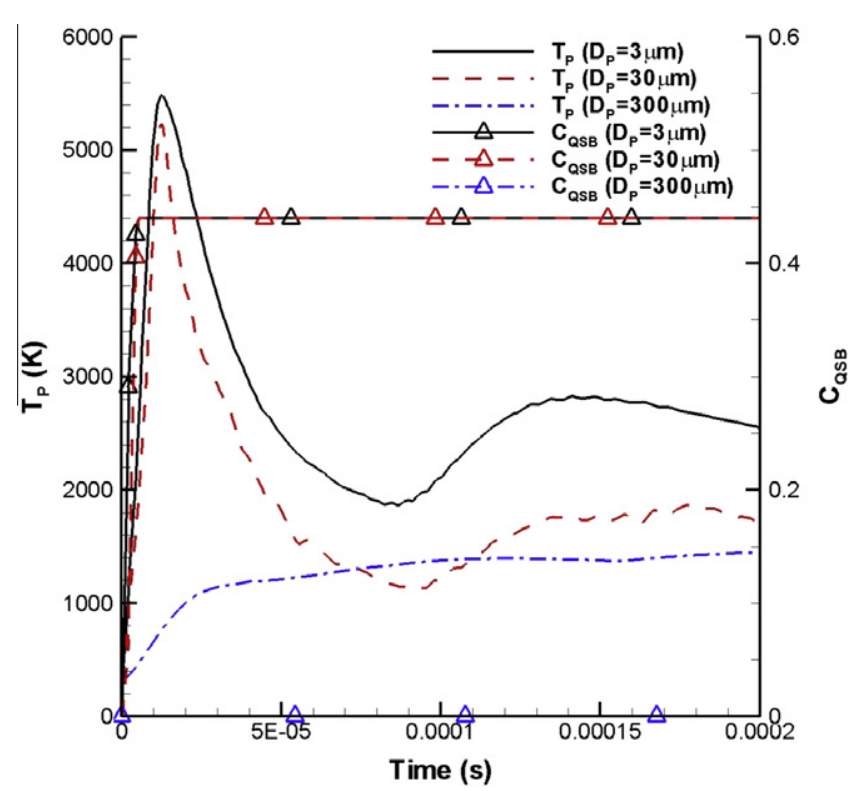

Fig. 24. Temperature and quasi-steady burning progress variable short time histories for Lagrangian tracer facing the elliptical end for initial particle diameters of $3 \mu \mathrm{m}, 30 \mu \mathrm{m}$, and $300 \mu \mathrm{m}$.

ing excellent agreement with the experimental data for the timing of the pressure waves and their magnitude resulting in good agreement to impulse measurements. Further cases are examined to determine the sensitivity of the mixture to initial charge equivalence ratio and particle diameter. As anticipated, smaller particles are preferable to maximize impulse from increasing the available area for burning, however, it should be noted that important dominate nanoscale effects have not be included (e.g., influence of oxide shell rupture on particle size). For the very large $300 \mu \mathrm{m}$ particles considered, the thermal inertia of particles resulted in considerable delay in heating relative to the time scales of the shock. For some of the particles, several cycles of melting followed by re-soldification is observed depending on the local shock reflection environment. 


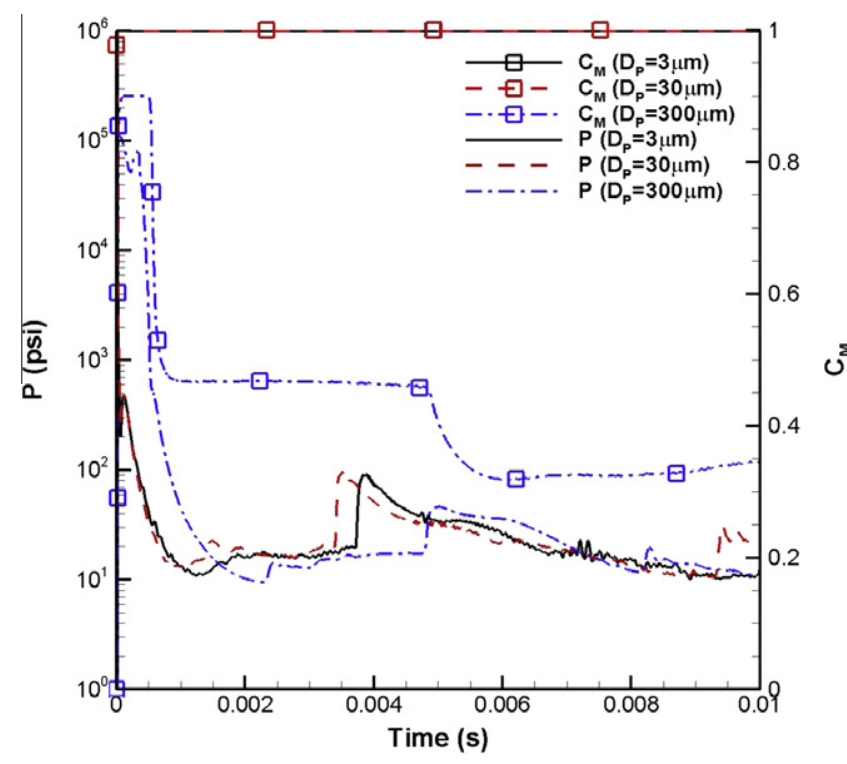

Fig. 25. Pressure and melting progress variable histories for Lagrangian tracer facing the chamber door for initial particle diameters of $3 \mu \mathrm{m}, 30 \mu \mathrm{m}$, and $300 \mu \mathrm{m}$.

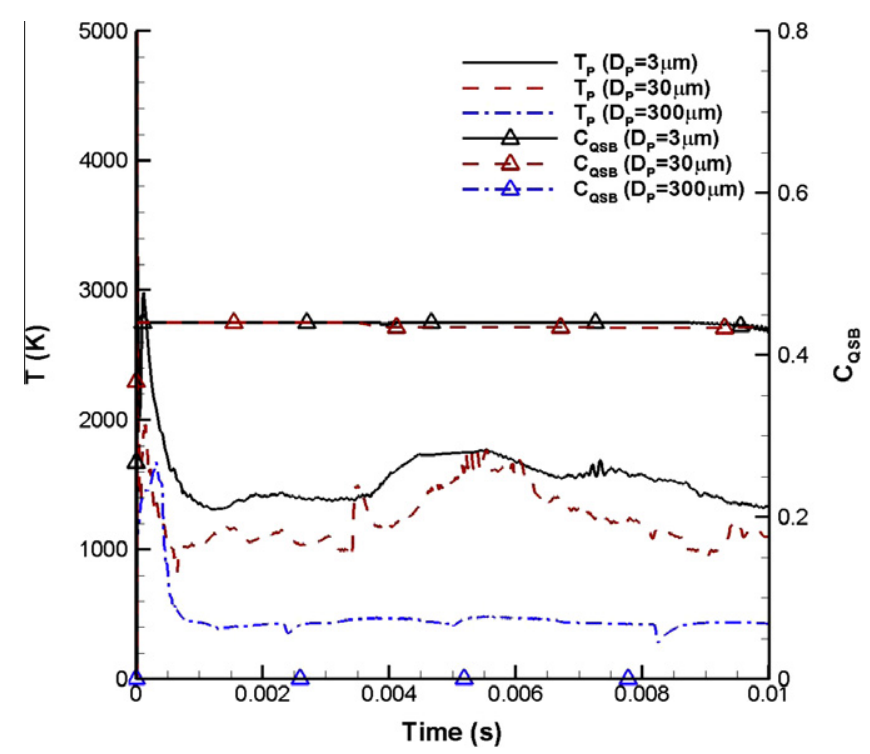

Fig. 26. Temperature and quasi-steady burning progress variable histories for Lagrangian tracer facing the chamber door for initial particle diameters of $3 \mu \mathrm{m}$, $30 \mu \mathrm{m}$, and $300 \mu \mathrm{m}$.

For marginally fuel rich charges $(1<\Phi<3)$ group combustion of the particles is observed as oxidizer left over from the detonation products is depleted and the particle begin to mix with the excess oxygen in the surrounding air. If the equivalence ratio is high enough, mixing of the surrounding oxygen into the fuel rich aluminum particulate is reduced resulting in a lower overall pressure. There is evidence of an optimal equivalence ratio to maximize pressure impulse around $\Phi=3$ for the geometry of the tank and initial conditions used. This optimal value is expected to depend on the initial charge configuration geometry and highlights the challenges in predicting the performance of non-ideal explosives.

\section{Acknowledgements}

The authors thank Dr. Robert Schmidt for his help implementing the multiphase model into CTH. Support for this work has been

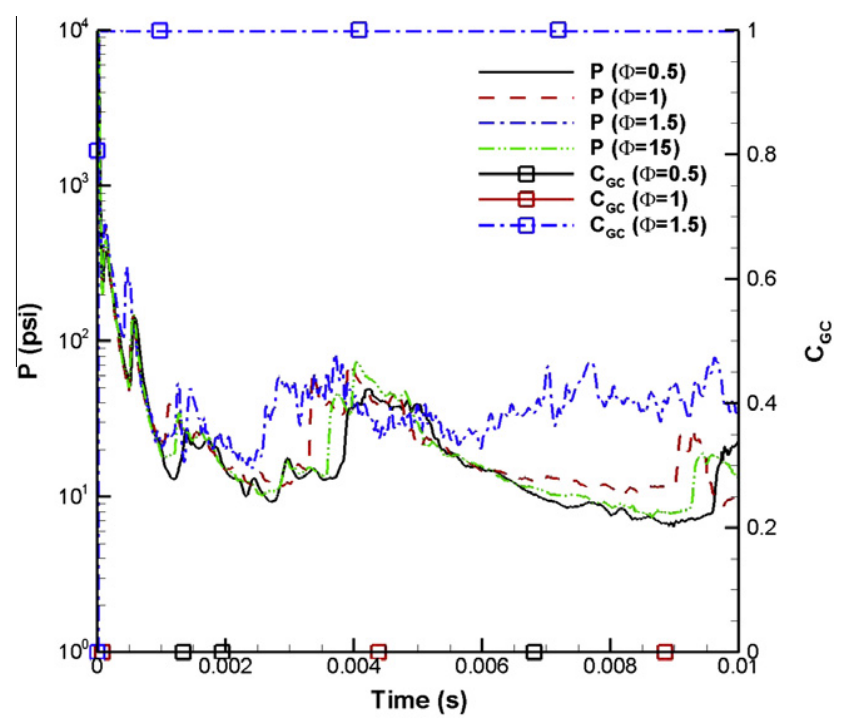

Fig. 27. Pressure and group combustion progress variable histories for Lagrangian tracer facing the elliptical end for $\Phi=0.5,1,1.5$, and 15 .

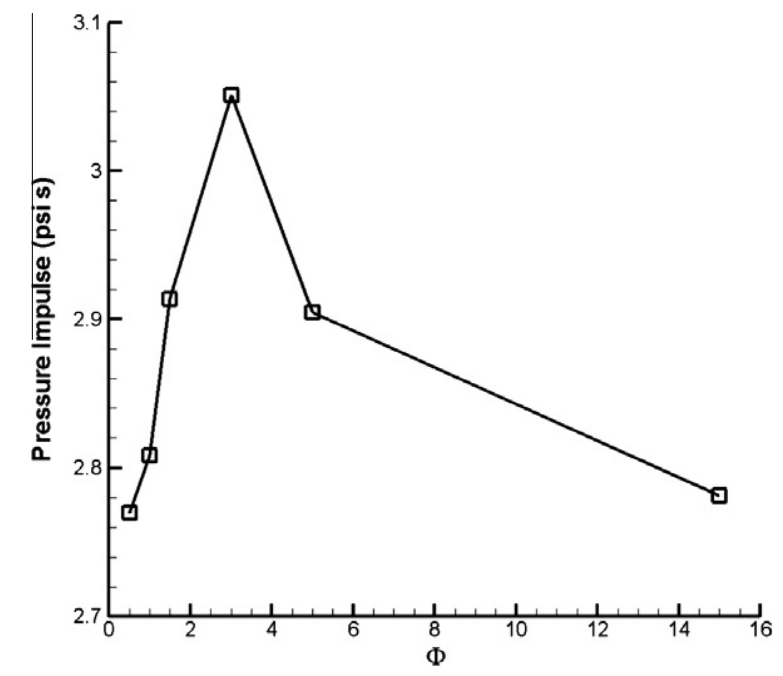

Fig. 28. Pressure impulse as a function of initial equivalence ratio for tracer history facing towards elliptical end of chamber.

provided by Sandia National Laboratories and the Department of Defense High Performance Computing Modernization Office. The second author also acknowledges the support of NAVAIR through the STTR phase I program (Contract N68335-10-C-0418).

\section{Appendix A. Pressure relaxation step}

The following algorithm is used to compute a pressure equilibrium state and the associated pressure work due to volume fraction changes. Assuming an adiabatic, non-reacting process the following ODE (Eq. (44)) is solved during this step:

$\rho_{s} \frac{d e_{s}}{d t}=-p_{I} \frac{d \alpha_{s}}{d t}$

where $p_{I}$ is the interface pressure which is approximated (Saurel et al., 2009) as, $p_{I}=\frac{c_{s} \rho_{s} p_{s}+c_{g} \rho_{g} p_{g}}{c_{s} \rho_{s}+c_{g} \rho_{g}}$, and $\alpha_{s}^{0}$ denotes the solid volume fraction at the beginning of the time step. Integrating Eq. (47) and assuming $\rho_{s}=\gamma_{s} \alpha_{s}$ is constant then, 
$e_{s}=e_{s}^{0}-\frac{1}{\rho_{s}^{0}} \int_{\alpha_{s}^{0}}^{\alpha_{s}} p_{I} \dot{\alpha}_{s} d t$

The integral can be discretized resulting in,

$e_{s}=e_{s}^{0}-\frac{p_{I}\left(\alpha_{s}-\alpha_{s}^{0}\right)}{\rho_{s}^{0}}$

An iterative approach is used to find the equilibrium pressure. Given an initial partial density for the solid and gas (i.e., $\rho_{s, g}^{0}=\alpha_{s, g}^{0} \gamma_{s, g}^{0}$ ), solid volume fraction, solid energy, mixture energy, and solid/gas pressures, an initial guess is made for the solid volume fraction change to achieve pressure equilibrium, $\Delta \alpha_{s}^{k+1}=\epsilon\left(\left(S_{P D}-1\right) \alpha_{s}^{k}+\right.$ $\left.\left(S_{P D}+1\right) \alpha_{g}^{k}\right) / 2$, where $S_{P D}=\operatorname{SIGN}\left(p_{s}^{0}-p_{g}^{0}\right)$ and $\epsilon$ is 0.5 . New solid and gas volume fractions are defined as, $\alpha_{s}^{k+1}=\alpha_{s}^{k}+\Delta \alpha_{s}^{k+1}$ and $\alpha_{g}^{k+1}=1-\alpha_{s}^{k+1}$. The solid and gas phase densities are then updated from $\gamma_{s}^{k+1}=\rho_{s}^{0} / \alpha_{s}^{k+1}$ and $\gamma_{g}^{k+1}=\rho_{g}^{0} / \alpha_{g}^{k+1}$. The change in solid energy due to pressure work is then updated using Eq. (49). The gas phase energy is then calculated from the mixture energy as, $e_{g}^{k+1}=\left(e^{0}-e_{s}^{k+1} Y_{s}\right) / Y_{g}$, where $Y_{s}$ and $Y_{g}$ are the mass fractions of the solid and gas phases, respectively. Using the new solid/gas densities and energies, a new pressure for the solid and gas phases are determined from the EOSs, $p_{s, g}^{k+1}=p_{s, g}\left(\gamma_{s, g}, e_{s, g}\right)$. A BKW EOS is used for the gas phase, assuming the composition is frozen during the adiabatic expansions/compression process, and SESAME tabular EOSs are used for the aluminum/alumina solid phases which are considered to be in pressure and temperature equilibrium. A separate Newton-Raphson method is used to evaluate the solid phase equilibrium state, which is described in Appendix B.

If $S_{P D}\left(p_{s}^{k+1}-p_{g}^{k+1}\right) \leqslant 0$ (i.e., the sign of the pressure difference has switched because too large of a solid volume fraction change is taken) the change in solid volume fraction is halved, ( $i$. e . $\Delta \alpha_{s}^{k+1}=\Delta \alpha_{s}^{k+1} / 2$ ) and new pressures are calculated for the new $\Delta \alpha_{s}^{k+1}$, as above. If the sign of the pressure difference is the same then the values for energy, density, pressure, and volume fractions at the $k$ state become the values at the $k+1$ state and the procedure is repeated. The algorithm is iterated until the convergence criteria, $\frac{\left|p_{s}^{k+1}-p_{g}^{k+1}\right|}{p_{s}^{k+1}+p_{g}^{k+1}} \leqslant 1 \times 10^{-10}$ is satisfied.

\section{Appendix B. Pressure and temperature equilibrium for solid phases}

The solid phase in the two-phase flow model is composed of aluminum and alumina which are assumed to be in pressure, temperature, and velocity equilibrium with each other. Both of their volume fractions are assumed to occupy the total solid volume fraction, $\alpha_{P}=\alpha_{\mathrm{Al}}+\alpha_{\text {mox }}$. The solid energy and temperature are defined as the mass weighted sums, $T_{P}=\left(Y_{\mathrm{Al}} T_{\mathrm{Al}}+Y_{\text {mox }} T_{\text {mox }}\right) /\left(Y_{\mathrm{Al}}\right.$ $\left.+Y_{\text {mox }}\right)$ and $e_{P}=\left(Y_{\mathrm{Al}} e_{\mathrm{Al}}+Y_{\text {mox }} e_{\text {mox }}\right) /\left(Y_{m}+Y_{\text {mox }}\right)$, respectively. The solid pressure is the volume weighted sum of constituents, $p_{P}=\alpha_{\mathrm{Al}} p_{\mathrm{Al}}+\alpha_{\text {mox }} p_{\text {mox }}$.

An iterative Newton-Raphson method is used to find the equilibrium state of the aluminum and alumina. Given an initial solid phase energy, mixture density, volume fraction, mass fractions, guessed solid phase temperature and guessed aluminum density, the aluminum volume fraction is $\alpha_{\mathrm{Al}}=\gamma_{\mathrm{Al}} / Y_{\mathrm{Al}} \rho$. The metal energy and pressure is determined from the EOS as a function of density and temperature. The metal oxide true density is then calculated from $\gamma_{\text {mox }}=Y_{\text {mox }} \rho /\left(\alpha_{P}-\alpha_{\mathrm{Al}}\right)$ and the metal oxide pressure and energy is found from the EOS. The guessed solid phase temperature and metal density are then updated via the iterative scheme until pressure and temperature equilibrium are achieved subject to the tolerances $\frac{\left|p_{A}^{k+1}-p_{\text {mox }}^{k+1}\right|}{p_{\mathrm{Al}}^{k+1}+p_{\text {mox }}^{k+1}} \leqslant 1 \times 10^{-10}$ for the pressure and $\left|T_{\mathrm{Al}}-T_{\text {mox }}\right|$ $\leqslant 1 \times 10^{-6}$. This iterative method is used each time the solid phase EOS is called.

\section{References}

Atkinson, A., 1985. Transport processes during the growth of oxide film at elevated temperature. Rev. Mod. Phys. 57, 437-470.

Baba, Y., Kurose, R., 2008. Analysis and flamelet modelling for spray combustion. J. Fluid Mech. 612, 45-79.

Babuk, V.A., Vasilyev, V., 2002. Model of aluminum agglomerate evolution in combustion products of solid rocket propellant. J. Propul. Power 18 $814-823$.

Baer, M.R., Nunziato, J.W., 1986. A two-phase mixture theory for the deflagrationto-detonation transition (DDT) in reactive granular materials. Int J. Multiphase Flow 12, 861-889.

Bazyn, T., Krier, H., Glumac, N., 2005. Oxidizer and pressure effects on the combustion of $10-\mu \mathrm{m}$ aluminum particles. J. Propul. Power 21, 577-582.

Bazyn, T., Krier, H., Glumac, N., 2007. Evidence for the transition from the diffusionlimit in aluminum particle combustion. Proc. Combust. Inst. 31, 2021-2028.

Bdzil, J.B., Menikoff, R., Son, S.F., Kapila, A.K., Stewart, D.S., 1999. Two-phase modeling of deflagration-to-detonation transition in granular materials: a critical examination of modeling issues. Phys. Fluids 11, 378-402.

Bellan, J., 2005. Perspectives on large eddy simulation for sprays: issues and solutions. Atomiz. Sprays 10, 409-425.

Benson, D.J., 1992. Computational methods in Lagrangian and Eulerian hydrocodes. Comput. Methods Appl. Mech. Eng. 99, 235-394.

Bilger, R.W., 1977. Reaction rates in diffusion flames. Combust. Flame 30, 277-284.

Bilger, R.W., 1993. Conditional moment closure for turbulent reacting flow. Phys. Fluids A 5, 436-444.

Bilger, R.W., 2000. Future progress in turbulent combustion. Prog. Energy Combust. Sci. 26, 367-380.

Bilger, R.W., 2011. A mixture fraction framework for the theory and modeling of droplets and sprays. Combust. Flame 158, 191-202.

Bray, K., Domingo, P., Vervisch, L., 2005. Role of the progress variable in models for partially premixed turbulent combustion. Combust. Flame 141, 431-437.

Brooks, K.P., Beckstead, M.W., 1995. Dynamics of aluminum combustion of metal particles. J. Propul. Power 11, 769-780.

Brzustowski, T.A., Glassman, I., 1964. Vapor-phase diffusion flames in the combustion of magnesium and aluminum - I: Analytical developments. Progress in Astronautics and Aeronautics, vol. 15. AIAA, New York, pp. 75-116.

Bucher, P., Yetter, R.A., Dryer, F.L., Parr, T.P., Hanson-Parr, D.M., Vicenzi, E.P., 1996. Flame structure measurement of single, isolated aluminum particles burning in air. Symp. (Int.) Combust. 26, 1899-1908.

Bucher, P., Yetter, R.A., Dryer, F.L., Vicenzi, E.P., Parr, T.P., Hanson-Parr, D.M., 1999 Condensed-phase species distributions about al particles reacting in various oxidizers. Combust. Flame 117, 351-361.

Carrara, M.D., DesJardin, P.E., 2006. A filtered density function approach to modeling separated two-phase flows using LES - I: Mathematical formulation. Int. J. Multiphase Flows 32, 365-384.

Carroll, M.M., Holt, A.C., 1973. Steady waves in ductile porous solids. J. Appl. Phys 44, 4388-4392.

Chang, C.-H., Liou, M.-S., 2007. A robust and accurate approach to computing compressible multiphase flow: stratified flow model and $\mathrm{AUSM}^{+}$-up scheme. J. Comput. Phys. 225, 840-873.

Chinnayya, A., Daniel, E., Saurel, R., 2004. Modeling detonation waves in heterogeneous energetic materials. J. Comput. Phys. 196, 490-538.

Chiu, H.H., Liu, T.M., 1977. Group combustion of liquid droplets. Combust. Sci. Technol. 17, 127-142.

Chiu, H.H., Kim, H.Y., Croke, E.J., 1982. Internal group combustion of liquid droplets. In: 19th Symposium (International) on Combustion, vol. 19, pp. 971-980.

Cooper, P., 1996. Explosives Engineering. Wiley-VCH, Inc., New York, NY.

Cowperthwaite, M., 1973. Tiger Computer Program Documentation. Tech. Rep., Stanford Research Institute.

DesJardin, P.E., 2005. Modeling of conditional dissipation rate for flamelet models with application to large eddy simulation of fire plumes. Combust. Sci. Technol. 177, 1881-1914.

DesJardin, P., Felske, J.D., Carrara, M.D., 2005. Mechanistic model for aluminum particle ignition and combustion in air. J. Propul. Power 21, 1-8.

Dreizin, E.L., 1996. Experimental study of stages in aluminum particle combustion in air. Combust. Flame 105, 541-556.

Dreizin, E.L., 1999. On the mechanism of asymmetric aluminum particle combustion. Combust. Flame 117, 841-850.

Dreizin, E.L., 2000. Phase changes in metal combustion. Prog. Energy Combust. Sci. 25, 57-78.

Drew, D.A., Passman, S.L., 1999. Theory of Multicomponent Fluids. Springer.

Fox, R.O., 2003. Computational Models for Turbulent Reacting Flows. Cambridge University Press, Cambridge, UK.

Fried, L., Souers, P., 1994. CHEETAH: A Next Generation Thermochemical Code. Tech. Rep., Lawrence Livermore National Laboratories.

Glassman, I., 1996. Combustion. Academic Press, New York, NY.

Hobbs, M.L., Baer, M.R., 1992. Nonideal thermoequilibrium calculations using a large product species data base. Shock Waves 2, 177-187.

Hultgren, R., Desai, P.D., Hawkins, D.T., Gleiser, M., Kelley, K.K., 1973. Selected Values of the Thermodynamic Properties of the Elements. American Society for Metals, Metals Park, Ohio.

Ihme, M., Pitsch, H., 2008. Prediction of extinction and reignition in nonpremixed turbulent flame using a flamelet/progress variable model 2: application in LES of SANDIA flames d and e. Combust. Flame 155, 90-107. 
Jackson, S.I., Kiyanda, C.B., Short, M., 2011. Experimental observations of detonation in ammonium-nitrate-fuel-oil (ANFO) surrounded by a high-sound-speed, shockless, aluminum confiner. Proc. Combust. Inst. 33, 2219-2226.

Kapila, A.K., Menikoff, R., Bdzil, J.B., Son, S.F., Stewart, D.S., 2001. Two-phase modeling of deflagration-to-detonation transition in granular materials: reduced equations. Phys. Fluids 13, 3002-3024.

Kerley, G., 1991. CTH Reference Manual: The Equation of State Package. Tech. Rep. Sandia National Laboratories.

Keshavarz, M.H., Mofrad, R.T., Poor, K.E., Shokrollahi, A., Zali, A., Yousefi, M.H., 2006. Determination of performance of non-ideal aluminized explosives. J. Hazard. Mater. 137, 83-87.

Krieger, F.J., 1970. The Thermodynamics of the Aluminum/Aluminum Vapor System. Technical Report RM-6335-PR, RAND.

Kumar, A., DesJardin, P.E., 2007. A reaction progress variable approach for LES of strongly radiating sooty flames. In: 5th Int. Seminar on Fire and Explosion Hazards. Fire \& Explosion Hazards. School of Engineering and Electronics, University of Edinburgh, pp. 425-434.

Kuo, K.K., 1986. Principles of Combustion. Wiley-Interscience.

Lallemand, M.H., Chinnayya, A., Metayer, O.L., 2005. Pressure relaxation procedures for multiphase compressible flows. Int. J. Numer. Methods Fluids 49, 1-56.

Law, C.K., 1973. A simplified theoretical model for the vapor-phase combustion of metal particles. Combust. Sci. Technol. 7, 197-212.

Liang, Y., Beckstead, M.W., 1998. Numerical simulation of unsteady, single aluminum particle combustion in air. AIAA

Ling, Y., Haselbacher, A., Balachandar, S., 2011a. Importance of unsteady contributions to force and heating for particles in compressible flows - Part 1: Modeling and analysis for shock-particle interaction. Int. J. Multiphase Flow 37, 1026-1044

Ling, Y., Haselbacher, A., Balachandar, S., 2011b. Importance of unsteady contributions to force and heating for particles in compressible flows - Part 2: Application to particle dispersal by blast waves. Int. J. Multiphase Flow 37, 1013-1025.

Liou, M-S. Chang C.-H. Nguyen, L. Theofanous, T.G. 2008. How to solve compressible multifluid equations: a simple, robust, and accurate method. AIAA 46, 2345-2356.

Mader, C.L., 1998. Numerical Modeling of Explosives and Propellants. CRC Press, Boca Raton, Fla.

Mathiesen, V., Solberg, T., Hjertager, B.H., 2000. An experimental and computational study of multiphase flow behavior in a circulating fluidized bed. Int. J. Multiphase Flow 26, 387-419.

McGlaun, J., Thomson, S., Elrick, M., 1990. CTH: a three-dimensional shock wave physics code. Int. J. Impact Eng. 10, 351-360.

Noh, W.F., Woodward, P., 1976. SLIC (simple line interface calculation). In: Proceedings of the Fifth International Conference on Numerical Methods in Fluid Dynamics. Lecture Notes in Physics, vol. 59. Springer, Berlin/Heidelberg, pp. 330-339.

Orth, L., Krier, H., 1998. Shock physics for nonideal detonations of metallized energetic explosives. Symp. (Int.) Combust. 27, 2327-2333.

Paillre, H., Corre, C., Garca Cascales, J.R., 2003. On the extension of the AUSM+ scheme to compressible two-fluid models. Comput. Fluids 32, 891-916.

Papalexandrix, M.V., 2005. Influence of inert particles on the propagation of multidimensional detonation waves. Combust. Flame 141, 216-228.

Petitpas, F., Massoni, J., Saurel, R., Lapebie, E., Munier, L., 2009. Diffuse interface model for high speed cavitating underwater systems. Int. J. Multiphase Flow 35 747-759.

Pierce, C.D., Moin, P., 2004. Progress-variable approach for large-eddy simulation of non-premixed turbulent combustion. J. Fluid Mech. 504, 73-97.
Pitsch, H., Ihme, M., 2005. An unsteady/flamelet progress variable method for les of nonpremixed turbulent combustion. Tech. Rep.

Pitsch, H., Cha, C.M., Fedotov, S., 2003. Flamelet modelling of non-premixed turbulent combustion with local extinction and re-ignition. Combust. Theory Model. 7, 317-332.

Radhakrishnan, K., Hindmarsh, A.C., 1993. Description and Use of LSODE, the Livermore Solver for Ordinary Differential Equations. Technical Report UCRLID-113855, Lawrence Livermore National Laboratory.

Ranz, W.E., Marshall, W.R., 1952. Evaporation from drops. Chem. Eng. Prog. 48, 141173

Reveilon, J., Vervisch, L., 2005. Analysis of weakly turbulent dilute-spray flames and spray combustion regimes. J. Fluid Mech. 537, 317-347.

Roberts, T.A., Burton, R.L., Krier, H., 1993. Ignition and combustion of aluminum/ magnesium alloy particles in $\mathrm{O}_{2}$ at high pressures. Combust. Flame 92, 125143.

Rosenband, V., 2004. Thermo-mechanical aspects of the heterogeneous ignition of metals. Combust. Flame 137, 366-375.

Ruggirello, K.P., DesJardin, P., Baer, M.R., Hertel, E.S., 2010. Modeling of particle compressibility and ignition from shock-shock focusing. Combust. Theory Model. 14, 41-67.

Samuelsberg, A., Hjertager, B.H., 1996. An experimental and numerical study of flow patterns in a circulating fluidized bed reactor. Int. J. Multiphase Flow 22, 575591.

Saurel, R., Abgrall, R., 1999. A multiphase Godunov method for multifluid and multiphase flows. J. Comput. Phys. 150, 425-467.

Saurel, R., Petitpas, F., Berry, R., 2009. Simple and efficient relaxation methods for interfaces separating compressible fluids, cavitating flows, and shocks in multiphase mixtures. J. Comput. Phys. 228, 1678-1712.

Saurel, R., Favrie, N., Petitpas, F., Lallemand, M.H., Gavrilyuk, S.L., 2010. Modelling dynamic and irreversible powder compaction. J. Fluid Mech. 664, 348-396.

Schtze, M., 2008. Fundamentals of High Temperature Corrosion. Wiley-VCH, pp. 67130.

Sirignano, W.A., 1999. Fluid Dynamics and Transport of Droplets and Sprays. Cambridge University Press, Cambridge, UK.

Smirnov, N.N., Zverev, N.I., Tyurnikov, M.V., 1996. Two-phase flow behind a shock wave with phase transitions and chemical reactions. Exp. Thermal Fluid Sci. 13, 11-20.

Stuhmiller, J.H., 1977. The influence of interfacial pressure forces on the character of two-phase flow model equations. Int. J. Multiphase Flow 3, 551-560.

Torquato, S., 2002. Random Heterogeneous Materials. Springer.

Truesdell, C., 1984. Rational Thermodynamics. McGraw Hill.

Turns, S.R., Wong, S.C., Ryba, E., 1987. Combustion of aluminum-based slurry agglomerates. Combust. Sci. Technol. 54, 299-318.

van Leer, B., 1979. Towards the ultimate conservative difference scheme. J. Comput. Phys. 39, 101-136

Washburn, E.B., Trivedi, J.N., Catoire, L., Beckstead, M.W., 2008. The simulation of the combustion of micrometer-sized aluminum particles with steam. Combust. Sci. Technol. 180, 1502-1517.

Washburn, E.B., Webb, J.A., Beckstead, M.W., 2010. The simulation of the combustion of micrometer-sized aluminum particles with oxygen and carbon dioxide. Combust. Flame 157, 540-545.

Widener, J.F., Liang, Y., Beckstead, M.W., 1998. Aluminum Combustion Modeling in Solid Propellant Environments. Tech. Rep.

Zhang, F., Thibault, P.A., Link, R., 2003. Shock interaction with solid particles in condensed matter and related momentum transfer. Proc. R. Soc. Lond. A Math. 459, 705-726. 